\title{
Eddies in motion: visualizing boundary-layer turbulence above an open boreal peatland using UAS thermal videos
}

\author{
Pavel Alekseychik $^{1,2}$, Gabriel Katul ${ }^{3,4}$, Ilkka Korpela ${ }^{5}$, and Samuli Launiainen ${ }^{1}$ \\ ${ }^{1}$ Bioeconomy and Environment, Natural Resources Institute Finland, 00790 Helsinki, Finland \\ ${ }^{2}$ Institute for Atmospheric and Earth System Research/Physics, Faculty of Science, \\ University of Helsinki, P.O. Box 68, 00014 Helsinki, Finland \\ ${ }^{3}$ Nicholas School of the Environment, Duke University, Durham, NC, USA \\ ${ }^{4}$ Department of Civil and Environmental Engineering, Duke University, Durham, NC, USA \\ ${ }^{5}$ Department of Forest Sciences, University of Helsinki, P.O. Box 27, 00014 Helsinki, Finland \\ Correspondence: Pavel Alekseychik (pavel.alekseychik@luke.fi)
}

Received: 17 September 2020 - Discussion started: 26 October 2020

Revised: 30 March 2021 - Accepted: 5 April 2021 - Published: 18 May 2021

\begin{abstract}
High-resolution thermal infrared (TIR) imaging is opening up new vistas in biosphere-atmosphere heat exchange studies. The rapidly developing unmanned aerial systems (UASs) and specially designed cameras offer opportunities for TIR survey with increasingly high resolution, reduced geometric and radiometric noise, and prolonged flight times. A state-of-the-art science platform is assembled using a Matrice 210 V2 drone equipped with a Zenmuse XT2 thermal camera and deployed over a pristine boreal peatland with the aim of testing its performance in a heterogeneous sedgefen ecosystem. The study utilizes the capability of the UAS platform to hover for prolonged times (about $20 \mathrm{~min}$ ) at a height of $500 \mathrm{~m}$ a.g.l. while recording high frame rate $(30 \mathrm{~Hz})$ TIR videos of an area of ca. $430 \times 340 \mathrm{~m}$. A methodology is developed to derive thermal signatures of near-ground coherent turbulent structures impinging on the land surface, surface temperature spectra, and heat fluxes from the retrieved videos. The size, orientation, and movement of the coherent structures are computed from the surface temperature maps, and their dependency on atmospheric conditions is examined. A range of spectral and wavelet-based approaches are used to infer the properties of the dominant turbulent scene structures. A ground-based eddy-covariance system and an in situ meteorological setup are used for reference.
\end{abstract}

\section{Introduction}

One of the long-standing problems in turbulence research, particularly turbulence in the planetary boundary layer (PBL), is the heat transfer between rough surfaces and the turbulent flow aloft. Eddies scour the surface and transport heat from the neighborhood of the roughness elements into the unobstructed flow. The precise nature of the eddies in terms of size and contact duration that effectively impinge and scour the surface to transport heat remains a formidable challenge and has been a subject of active research for several decades (Owen and Thompson, 1963; Adrian, 2007). With rapid advancements in thermal infrared (TIR) imaging and image processing, a new arsenal of experimental methods pave the way to progress on these issues, which motivates the present work.

This study focuses primarily on the properties of large coherent turbulent structures, or dominant eddies as termed by Taylor (1958). He was the first to draw attention to the regular features in air temperature time series, which Priestley (1959) later linked to the thermals generated by surface roughness and buoyancy. Air parcels residing near the ground attain buoyancy upon receipt of heat from the ground and rise up to become replaced by cooler air parcels descending from above in a cyclical manner. Such ascending and descending air parcels can reach the size of the entire boundary layer, i.e., hundreds to thousands of meters across (Kaimal and Businger, 1970; Kaimal et al., 1976). 
However, such a mechanistic view gradually evolved into an extensive theory describing the coherent structures in increasingly higher detail. The early research mostly relied on anemometer arrays and provided often conflicting evidence about the organization of PBL coherent structures. The cross section, average non-dimensional temperature, and diameter of a thermal strongly depend on stability, according to Frisch and Businger (1973). Work by Khalsa (1980) and by Wilczak and Tillman (1980) confirmed a decrease in plume length with increasing instability. In contrast, Antonia et al. (1979) found much less consistency in the stability effect. Kaimal (1974) observed plumes that travel as constant entities with the same velocity at all heights, their translation velocity being less than the mean wind speed. However, Wilczak and Tillman (1980) present translation velocities that are always greater (1.13 times, on average) than the mean wind, although there was a large scatter in plume translation velocities (tall plumes traveling faster than short ones); translation directions often deviated from the mean wind direction. Kaimal et al. (1976) observed plumes merging and forming boundary layer-scale structures, while Webb (1977) demonstrated larger-scale coherent structures he termed "thermal walls" translating at the velocity of the mean wind and much smaller thermals confined to the low altitudes that did not interact with the "walls". In moderately unstable conditions, observations were made of plume elongation with length/width ratios of 4-12 (Davison, 1975). Wilczak and Tillman (1980) report the typical lengths of $300 \mathrm{~m}$, widths of $40 \mathrm{~m}$, and length/width ratios of 5-10.

The visualization of the coherent turbulent structures underwent a long evolution. Their exact shape was first demonstrated by particle image velocimetry (PIV) done on wind tunnel measurements. Streamwise streakiness of the wall-bounded flow velocity field was first shown by Kline et al. (1967). The PIV experiments by Tomkins and Adrian (2003) and by Ganapathisubramani et al. (2003) revealed a structure consisting of low- and high-momentum regions elongated along the wind direction and measured 1020 times the boundary layer depth. Kim and Adrian (1999) and Guala et al. (2006) referred to them as very large-scale motions (VLSMs), and Kim and Adrian (1999) proposed that they consist of hairpin vortex packets. As the PIV setups of the previous studies limited the horizontal domain to about 2 times the boundary layer depth, the large-scale streakiness and underlying hairpin structure of the VLSMs could only be shown to their full extent by direct numerical simulation (DNS) of wall-bounded turbulent flows (e.g., Jeong et al., 1997) and large eddy simulation (LES) of atmospheric surface layers (e.g., Fang and Porté-Agel, 2015).

Estimating the size, shape, motion, and timescales of such coherent structures under real PBL conditions remains a difficult task. The principal applicability of TIR to turbulence studies has been established in several field studies. Some of the earliest experiments (Hoyano et al., 1999; Sugawara et al., 2001; Chudnovsky et al., 2004; Meier et al., 2011) used
TIR in an urban setting to determine the thermal properties of various surfaces and to remotely estimate the components of the surface energy balance. Vogt (2008) was the first to record thermal videos over a grass field, which was soon followed by Garai and Kleissl (2011) and a similar experiment to describe the temporal skin temperature variation on various urban surfaces (Christen and Voogt, 2009). Those studies established the possibility of visualizing and analyzing the different scales of turbulent eddies. Garai and Kleissl (2011) reported that the largest of the coherent structures were apparently much bigger than the patch of ground they measured and proposed that flying a thermal camera suspended on a balloon at a few hundred meters above ground would improve detection of larger eddies. Modern multirotor UASs are capable of efficiently performing this task.

None of the previous TIR experiments covered a surface area exceeding ca. $200 \mathrm{~m}$ in diameter. The largest-scale outdoor TIR surface experiment so far is that of Garai and Kleissl (2013), in which an area $275 \times 207 \mathrm{~m}$ was imaged. A similar approach by Christen et al. (2012) used a camera with a very oblique view angle targeted at a complex urban environment, which, in the presence of a highly irregular shape of the underlying surface, precluded any spatial study of turbulence. Inagaki et al. (2013) and Morrison et al. (2017) recorded TIR sequences at frequencies exceeding $30 \mathrm{~Hz}$, but measured relatively small areas of about $15 \times 3 \mathrm{~m}$ and $5 \times 2 \mathrm{~m}$, respectively. The previous studies are, therefore, characterized by low spatial coverage, and disadvantageous positioning of the camera resulting in large view-zenith angles (mounting close to the ground with an oblique view angle) - all of which are alleviated by the UAS approach employed in the present study.

In this work, we explore the capability of UAS thermal imagery for detecting variations in surface temperature at high spatial and temporal resolution. Generally, we aimed to demonstrate that the near-nadir thermal imagery used here can enable inquiry into the particularities of the coherent structures' evolution and movement deduced from their 2D thermal traces on the ground. The specific goals are (1) to test the applicability of UAS TIR imagery for near-surface turbulence studies and to develop the necessary methodology to correct and analyze the images; (2) to describe the time and length scales of the entire spectrum of surface temperature that is responsive to eddy impingement, with a focus on large structures; and (3) to compare the UAS-based turbulence characteristics to those measured by ground-based sonic anemometry.

A two-day field experiment over a pristine boreal peatland in southern Finland was conducted using the thermal/RGB camera mounted on an unmanned quadcopter. This site was selected for two pragmatic reasons: the presence of shortstature vegetation with low thermal inertia, so as to minimize the so-called "honami" effect, and due to the available eddy-covariance (EC) tower measurements and meteorological data. 


\section{Materials and methods}

\subsection{Thermal imaging by the UAS}

The principal sensor used was a thermal/RGB camera DJI Zenmuse XT2 mounted on a DJI Matrice 210 v2 quadcopter (Fig. 1). The IR sensor of the XT2 camera is a FLIR Tau 2 (FLIR Systems Inc.). The FLIR Tau 2 sensor measured in the 7.5-13.5 $\mu \mathrm{m}$ range and had a resolution of $640 \times 512$ pixels. The view angle of the XT2 was $45^{\circ} \times 37^{\circ}$ with a $13 \mathrm{~mm}$ lens. The thermal resolution was better than $<0.05 \mathrm{~K}$ and the maximum sampling frequency was $30 \mathrm{~Hz}$.

Retrievals of IR videos were conducted in four flights near noon, two on 6 August and two on 28 August 2019, at the Siikaneva pristine boreal peatland complex in southern Finland. Clear sky conditions prevailed during all four flights. About $5 \mathrm{~min}$ thermal camera warmup time was allowed between UAS power-on and takeoff, as longer warmup is impractical considering the limited battery life. In each flight, the drone hovered above an EC tower at an altitude of $500 \mathrm{~m}$ and was able to automatically maintain the position irrespective of the wind. However, the rotation about the downlooking optical axis required manual correction throughout the flight as the drone tended to slowly turn while hovering. However, this issue was of minor importance as any such rotation is efficiently corrected at the image registration step.

The first three $30 \mathrm{~Hz}$ TIR video retrievals lasted for about $20 \mathrm{~min}$ (Table 1), whereas the fourth retrieval was $10 \mathrm{~min}$ due to a gimbal malfunction. The longest possible hovering flight time of $20 \mathrm{~min}$ matches the producer's estimate of battery life including the additional $6 \mathrm{~min}$ reserved for ascent and descent. A TIR sequence of 20 min thus approaches the conventional $30 \mathrm{~min}$ averaging period for computing turbulence statistics and vertical heat fluxes from ground-based sonic anemometry. Immediately before each flight, a nonuniformity correction was performed for the thermal camera. This correction introduces large step-changes in the measured temperature field, which are also non-uniform across the image. It was not applied again during the flight as this would have been detrimental for the detection of frame-toframe temperature differences at high spatial and temporal resolution. To synchronize the drone thermal video with local time (and thus the EC record time), a reference signal was created at an arbitrary moment in each flight by quickly moving a $20 \times 60 \mathrm{~cm}$ aluminum plate and by recording the exact time of this manipulation. The plate otherwise lied static on the ground for the duration of the flight. The movement of the plate was easily detected in the thermal sequences recorded from a $500 \mathrm{~m}$ altitude. As a result, the UAS and EC data sets were synchronized at an error of less than $1 \mathrm{~s}$.

During each 20 min flight, the XT2 camera recorded surface temperature at $30 \mathrm{~Hz}$, producing $20 \mathrm{~GB}$ of raw data in the FLIR file format. The sequences were, therefore, sub-sampled to $1 \mathrm{~Hz}$ as a means of reducing data set size and processing times, while preserving the relevant tur- bulence timescales. The surface emissivity was set to a constant of 0.98 , as the actual emissivities of the peatland surface constituents are not precisely known; however, they are expected to be about 0.98 as a representative value of a moss-dominated boreal ecosystem emissivity (Antti-Jussi Kieloaho, personal communication, 2020). Before further analyses, the sequences were converted into MATLAB $^{\circledR}$ data arrays (.mat) using the FLIR ResearchIR ${ }^{\circledR}$ software; MATLAB ${ }^{\circledR}$ was used for further data processing. ResearchIR also performed the correction for the transmissivity in the $500 \mathrm{~m}$ atmospheric column between the drone and the ground using mean air temperature and relative humidity observed during the retrievals.

\subsection{Data post-processing}

The data post-processing workflow in Fig. 2 consists of sequential steps from handling the raw sequences to inferring the impingement of turbulent motions from surface temperature data. Steps 1-4 can be considered to be common for all UAS-based thermal video surveys, while steps 5-6 are related to the specific aim of retrieving turbulence characteristics.

\subsubsection{Vignetting correction}

The vignetting effect is the artificial radial reduction in brightness temperature away from the image center, resulting from the varying lens transmissivity. A method was developed to define this lens-specific artifact and remove the effect from each image. Before each flight, an image was taken of a plane fabric surface painted black to serve as a homogeneous temperature field. The reduction in temperature in the corners versus the center region of this thermogram $(100 \times 100$ pixels) was found to exceed $2 \mathrm{~K}$. While the effect proved to be non-symmetrical with respect to the center of the image, it was well described by a fourth-degree polynomial surface fit (Appendix A).

\subsubsection{Geometric calibration}

A practical approach was developed and implemented for geometric calibration of the TIR camera. A checkerboard similar to what is commonly used for RGB camera calibration was constructed using $5 \mathrm{~cm}$ paper squares glued onto an aluminum plate (see Appendix B). The aluminum plate was heated up on a stove to increase the contrast between the high-emissivity paper checkers and the low-emissivity aluminum checkers. A set of 28 images of the checkerboard taken at different angles and distances was fed to the MATLAB ${ }^{\circledR}$ camera calibrator tool, which yielded the camera intrinsic parameters (Appendix B). 

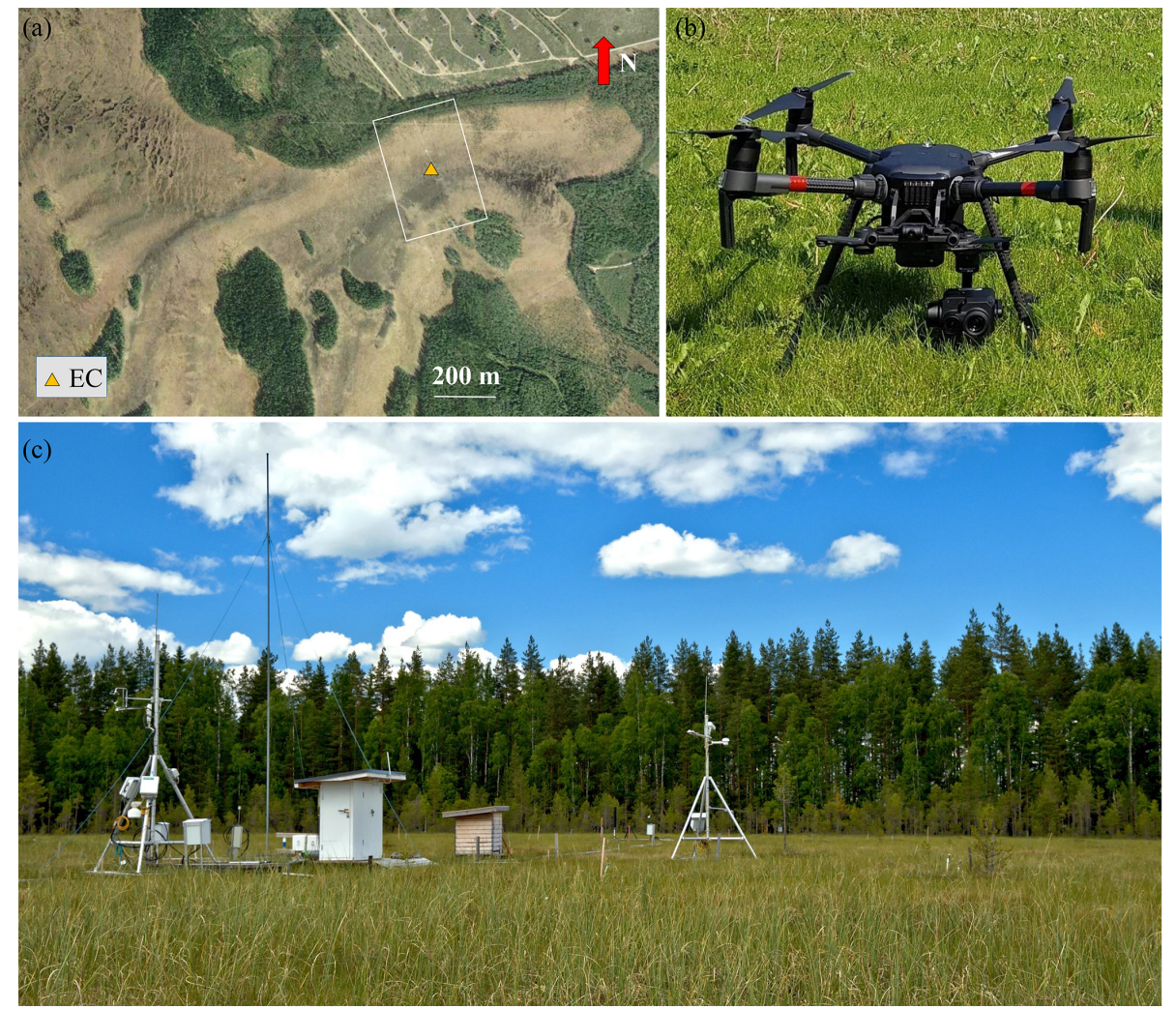

Figure 1. (a) Satellite view of the region surrounding the measurement site $\left(61.832^{\circ} \mathrm{N}, 24.193^{\circ} \mathrm{E}\right)$. The area imaged by the drone is shown with the white box. A (C) Google Earth screenshot is used. (b) The UAS consisting of a DJI Matrice 210 v2 drone and a DJI Zenmuse XT2 thermal/RGB camera. (c) Siikaneva fen ICOS site captured at the peak of the sedge leaf area, viewed towards the northwest. The EC tower is on the extreme left.

Table 1. Flight metadata. The times specified are UTC +3 .

\begin{tabular}{lllllr}
\hline Date & Altitude & $\begin{array}{l}\text { Start } \\
\text { time }\end{array}$ & $\begin{array}{l}\text { End } \\
\text { time }\end{array}$ & $\begin{array}{l}\text { Total } \\
\text { duration }\end{array}$ & $\begin{array}{r}\text { 1 Hz frame } \\
\text { count }\end{array}$ \\
\hline 6 August 2019 & $500 \mathrm{~m}$ & $12: 28: 54$ & $12: 47: 58$ & 19 min 4 & 1144 \\
6 August 2019 & $500 \mathrm{~m}$ & $13: 28: 35$ & $13: 47: 17$ & 18 min 42 $\mathrm{s}$ & 1122 \\
28 August 2019 & $500 \mathrm{~m}$ & $11: 22: 24$ & $11: 42: 16$ & 19 min 52 $\mathrm{s}$ & 1193 \\
28 August 2019 & $500 \mathrm{~m}$ & $12: 23: 09$ & $12: 33: 36$ & 10 min 27 s & 629 \\
\hline
\end{tabular}

\subsubsection{Image registration}

The small, but significant, motion of the UAS during the imaging means the images needed to be co-registered, i.e., rotated and translated to a common coordinate system to ensure that each pixel in the output thermal video corresponds to the same point on the ground. The first frame of each of the four TIR sequences was selected as a reference, with all the subsequent frames being co-registered with it. A satisfactory solution was achieved using an intensity-based approach using the imregister MATLAB ${ }^{\circledR}$ function. Parameterization was as follows: optimization configuration $=$ OnePlusOneEvolutionary, MattesMutualInformation $=$ True, maximum iterations $=300$, and initial ra- dius $=0.001$. With these settings, imregister performed an iterative solution of the image registration problem using the $(1+1)$ evolutionary approach using Mattes Mutual Information as a criterion of similarity between the moving and reference images. The $(1+1)$ evolutionary optimizer involves the generation of perturbed images based on a Gaussian probability function. The perturbed image versions more similar to the template are kept, while the less similar are rejected (Styner et al., 2000). The process is continued until convergence between the perturbed and template images is achieved. Mutual Information combines the joint entropy between the images and their individual entropies as a measure of their statistical relationship; Mattes Mutual Information 


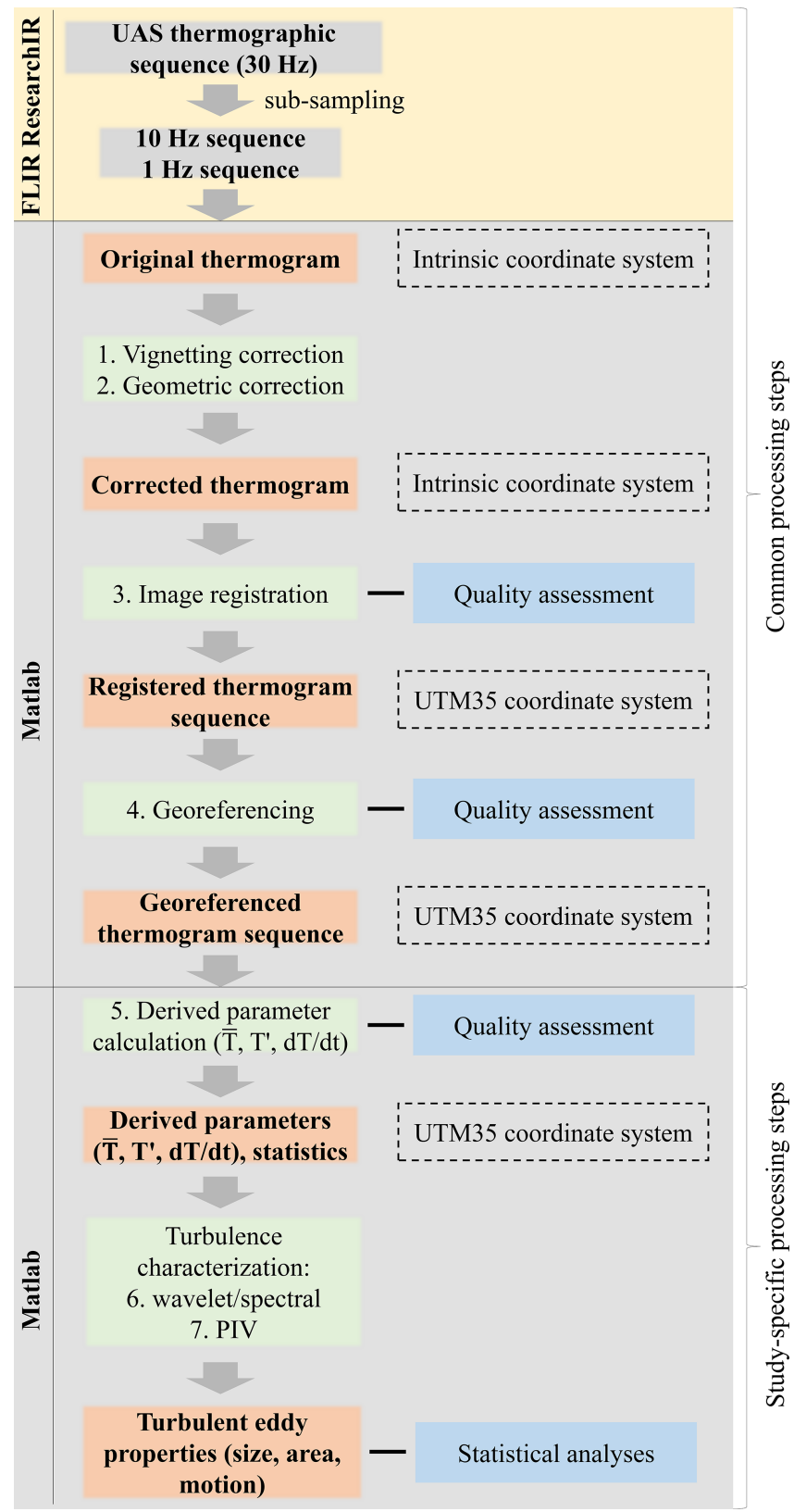

Figure 2. Data processing workflow. The workflow is divided into the stages executed with ResearchIR and MATLAB. Common processing steps are of general applicability to thermal video analysis, while the study-specific steps serve the aims of the current work. The processing steps numbered 1-7 are shown in green boxes and their output in orange boxes. Quality assessment steps and the coordinate system used at each step are shown in the righthand column.

uses a single set of pixel locations instead of generating it at each iteration (Mattes et al., 2001).

The quality of registration was evaluated for the pairs $i$ th image-reference image (the first image of the sequence) using the structural similarity index measure (SSIM), mean squared error (MSE), and peak signal-to-noise ratio (PSNR) as metrics (Appendix C). When the registration algorithm failed to converge (typically only a few images per flight), SSIM and PSNR displayed a downward peak and MSE an upward peak (not shown). The $i$ th image that could not be registered was replaced with the $(i-1)$ th image.

\subsubsection{Georeferencing}

The registered images were georeferenced in order to spatially relate them to the EC tower and to the georeferenced UAS RGB photo. Four ground control points (GCPs) in the form of $2 \times 2 \mathrm{~m}$ crosses with $20 \mathrm{~cm}$ wide arms were constructed from aluminum sheets. The GCPs formed an irregular quadrilateral with the corners at approximately $100 \mathrm{~m}$ distance from the EC tower. The UTM35 coordinates and ellipsoidal heights of GCPs were measured using a kinematic GNSS device (Trimble Catalyst DA1) at centimeterlevel accuracy. The ground sampling density of the image pixels is $0.6 \mathrm{~m}$ resulting in blurred images of the crosses; the GCP was, therefore, determined by searching for local interpolated temperature minima within a small search area (ca. $10 \mathrm{~m}$ across) of the expected location of the aluminum targets. Owing to large differences in emissivity, the GCP pixels were seen in strong contrast, thereby enabling georeferencing with a small sub-pixel level RMS error (Appendix D). The center of the georeferenced images has a UTM latitude of 6858732 and longitude of 352185 (UTM zone 35V). The origin $(0,0)$ of the images corresponds to a UTM latitude of 6858485 and longitude of 351968.

\subsubsection{Derived parameters, averaging, and notation}

The Cartesian coordinates used are $(x, y, z)$ with $x$ being the longitudinal (or along mean wind) direction, $y$ being lateral, and $z$ being the vertical direction with $z=0$ being the ground surface. The three instantaneous velocity components $(u, v, w)$ are aligned along the $x-, y$-, and $z$-axes, respectively. Because the work here uses different averaging procedures including time (e.g., variables sampled at the EC tower), space, and space-time, the following conventions are used to indicate the averaging operators for an arbitrary flow variable $\chi$ evolving in space $(x, y)$ and time $(t)$. Time averaging (taken over the flight duration) at a given location $(x, y)$ is indicated by overlines $\bar{\chi}$ and deviations from time averaged quantities are indicated by primes so that $\chi(x y t)=\bar{\chi}(x y)+\chi^{\prime}(x y t)$. Spatial averaging (over the sampled image domain) at a given $t$ is indicated by brackets $\langle\chi\rangle$ and deviations from this spatial average are indicated by double primes so that $\chi(x y t)=\langle\chi\rangle(t)+\chi^{\prime \prime}(x y t)$. Space-time averaging (over the image domain and flight duration, i.e., the overall mean temperature recorded during the flight) is indicated by a hat $\hat{\chi}$ and deviations from this space-time average are indicated by a tilde so that $\chi(x y t)=\hat{\chi}+\tilde{\chi}(x y t)$. For the instantaneous georeferenced surface temperature field $T(x, y, t)$, a space-time average 
was applied so that $\tilde{T}(x y t)=\hat{T}-T(x y t)$. A time averaging at each pixel location was then conducted to obtain $\overline{\tilde{T}}(x y)$ such that $T^{\prime}(x, y, t)=\tilde{T}(x y t)-\overline{\tilde{T}}(x y)$. Such a "zeroing" was designed to minimize the artificial changes in recorded absolute temperature due to the drift of the FLIR Tau 2 thermal sensor on the Zenmuse XT2 (Dugdale et al., 2019). Temperature fluctuation distributions (see Fig. 5 and discussion therein) revealed that the physically sound $T^{\prime}(x, y, t)$ values were contained in the interval $-1.5<T^{\prime}<1.5 \mathrm{~K}$, whereas the more extreme values are deemed to represent noise; the $T^{\prime}(x, y, t)$ was, therefore, de-spiked using those threshold bounds.

\subsubsection{Characterizing turbulent eddy size and shape: spectral and wavelet analysis}

The post-processed $T^{\prime}(x, y, t)$ are used to characterize the boundary-layer eddies impinging on the surface. Their spectral properties are featured first, followed by the transport patterns (i.e., imprint of advection velocity of large coherent eddies), size, and area. A comparison between spacetime surface temperature and high-frequency air temperature measured at the EC location is conducted. Lastly, implications of $T^{\prime}(x, y, t)$ to the determination of sensible heat flux from modified flux-variance similarity are discussed. Power spectra of $T^{\prime}(x, y, t)$ were derived from both the drone maps and the EC-based sonic temperature. The power spectra were calculated in both the temporal and 2D spatial domains using fast Fourier transform (FFT).

Additionally, we used wavelet transform to infer 2D power spectra (MATLAB ${ }^{\circledR}$ Wavelet Analysis Toolbox) and segregate the individual large coherent structures. The Mexican Hat wavelet was applied to decompose the sequences at the spatial scales of 1-50 m; such a range was chosen based on the assumption that the maximum eddy size well represented by a TIR image covering ca. $340 \times 430 \mathrm{~m}$ would be roughly $100 \mathrm{~m}$. A 2D wavelet transform was then applied to the $T^{\prime}(x, y, t)$ sequences to characterize the larger coherent turbulent structures in the following manner. A $14 \mathrm{~m}$ scale was chosen for this particular purpose based on visual evaluation of how well the large coherent structure boundary was delineated; a transform at this scale yielded isolated regions that best matched the most pronounced thermal traces. It must be noted that the wavelet transform scale is a sensitive parameter requiring adjustment to the scale of the dominant eddies; an excessively small scale value would lead to erroneous division of large eddies, while a scale value that is too high would result in grouping where the eddies are apparently separate. A 2D wavelet transform was applied to each image of the $T^{\prime}(x, y, t)$ sequences, with the pixels having wavelet powers smaller than -3.5 or greater than 3.5 set to $\mathrm{NaN}$ to enhance the contrast between the positive and negative wavelet power regions. The threshold for this filtering operation should also be chosen with care, as the slopes sep- arating positive and negative wavelet regions can be steep (see the effect of the \pm 3.5 threshold in Fig. $3 \mathrm{c}$ ). The positive and negative regions remaining after that filtering operation represent, in essence, the smoothed boundaries of the larger coherent structure thermal traces. The wavelet transform image was discretized by setting positive regions to 1 and negative to -1 , after which they were labeled by watershed transform. The labeled regions were then filtered by area (restricted to 500-50000 $\mathrm{m}^{2}$ ) and mean absolute value of $T^{\prime}(x, y, t)$ within their boundaries $\left(T^{\prime}(x, y, t)\right.$ must be $>0.06 \mathrm{~K})$. Finally, the MATLAB function regionprops was applied to extract the minor axis (width), major axis (length), orientation, area, and the mean $T^{\prime}(x, y, t)$ of each region. The regions now represent the boundaries of large coherent structures. These operations were performed on each image of the $1 \mathrm{~Hz} T^{\prime}(x, y, t)$ sequences; Fig. 3 gives a visual example of the above operations.

Another approach to spectral analysis was taken by calculating the mean of along- and cross-wind FFT spectra. To do so, each image was first rotated so as to make rows aligned with the anemometer wind direction (WD) averaged within \pm 30 s of the image's time stamp. Then, the spectra were calculated for the rows and columns and averaged, yielding the along- and cross-wind spectra, respectively. The rows and columns containing less than $3001 \mathrm{~m}$ values after rotation were omitted from the calculation. FFT was also applied to the thermal sequences in the temporal domain. FFT was first performed on individual pixel time series, and then those pixel-wise spectra were averaged to yield a single FFT spectrum of a flight.

\subsubsection{Characterizing turbulent eddy advection velocity: particle image velocimetry}

An open-source tool PIVlab (Thielicke and Stamhuis, 2014) was used for thermal image velocimetry (TIV) processing to derive the speed and direction of the coherent structures motion. TIV processing was performed on the $T^{\prime}$ maps wavelet transformed at the scale of $5 \mathrm{~m}$, which provided the necessary de-noising. TIV yielded the horizontal velocity vector field of the coherent turbulent structure motions, from which the image-average advection velocity and direction were also derived. The background component of the images was removed using a built-in PIVlab GUI. After a series of tests, the following settings were chosen: interrogation area: 100 pix (i.e., $100 \mathrm{~m}$ at $1 \mathrm{~m}$ per pix); step: 50 pix; sub-pixel estimation method: Gauss 2x3; correlation quality: extreme; autocorrelation: disabled. As PIVlab analyzes pairs of images, this leads to a new wind field calculated for each $1 \mathrm{~Hz}$ image pair, i.e., once every $2 \mathrm{~s}$. The output wind vectors were filtered with the threshold of $3 \sigma$ in order to remove the outliers. For presentation in the case studies (Sect. 3.4), the TIV wind fields was averaged over a period of $80 \mathrm{~s}$ (see below), which provided extra smoothing. Being under $1 \mathrm{~m}$, the spatial errors introduced in image registration and georeferencing do 

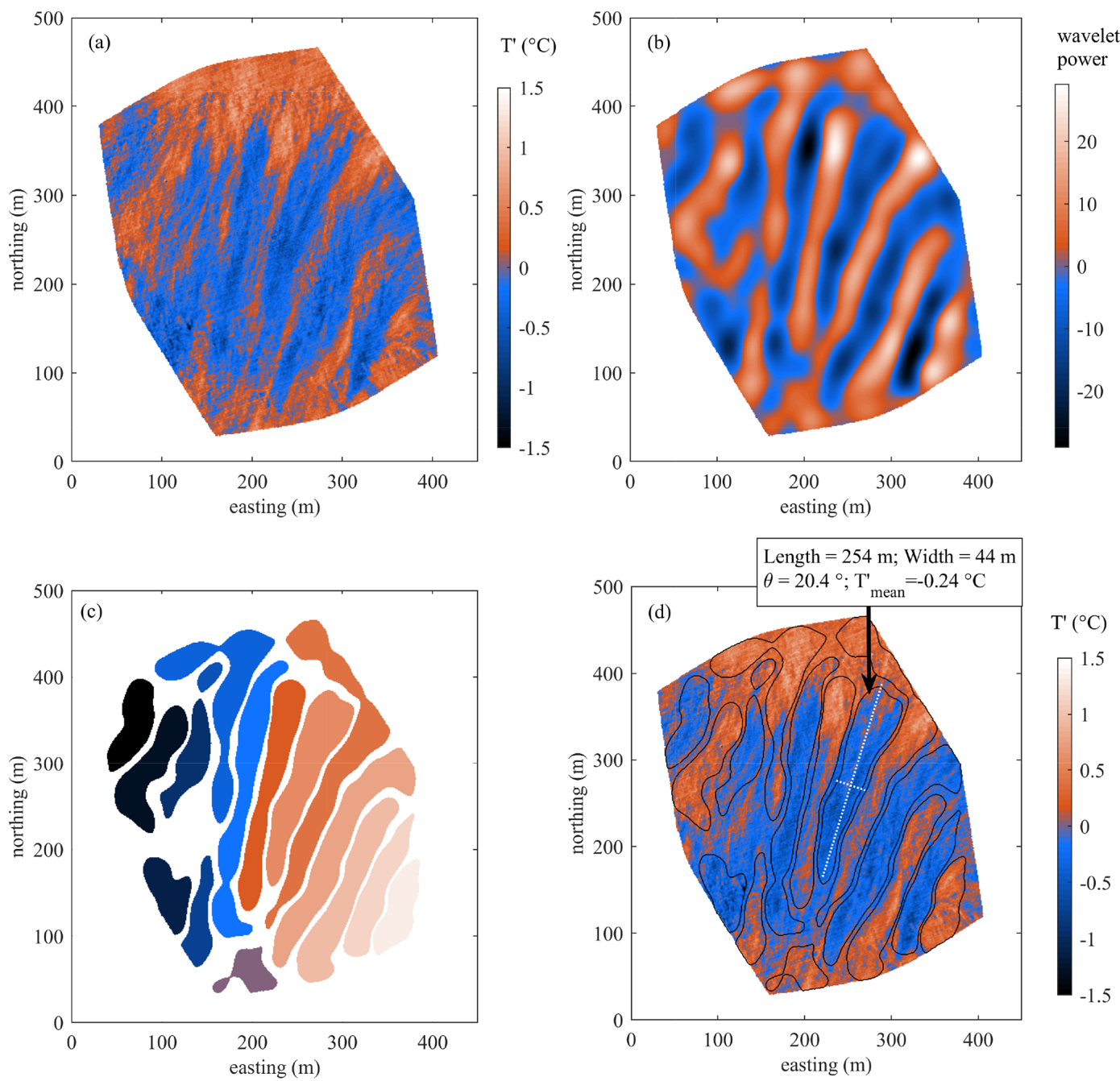

Figure 3. Large coherent structure identification method. (a) Original georeferenced $T^{\prime}(x, y, t)$ image, (b) $2 \mathrm{D}$ wavelet transform at $14 \mathrm{~m}$ scale, (c) labeled coherent structure thermal imprints obtained by watershed transform of (b), and (d) original $T^{\prime}(x, y, t)$ image (a) shown with the coherent structure boundaries from (c) and detailed information given for one of the identified structures (length, width, clockwise rotation from the vertical, and mean temperature excursion within its boundary). The colors in (c) are only to tell the structures apart and do not correspond to the color bars in the other panels.

not influence the mean TIV wind fields. However, we find that the scale of wavelet transform applied to the input images does; the best performance is achieved with evenly distributed, small, and numerous "particles" (or thermal traces in the case of TIV), which motivated the choice of the $5 \mathrm{~m}$ wavelet decomposition scale. By trial and error, we established that a smaller scale leads to insufficient smoothing, which distorts the TIV output, whereas a higher scale addresses the movement of large eddies, which are sparser and more difficult to process into a continuous wind field.

\subsection{Ground-based measurements}

Turbulent wind components and sonic temperature, as well as incoming global radiation and air humidity and temperature were measured on the EC tower $\left(61^{\circ} 49^{\prime} 57.324^{\prime \prime} \mathrm{N}\right.$, $24^{\circ} 11^{\prime} 34.116^{\prime \prime}$ E) of the Siikaneva fen ICOS ecosystem monitoring station. The measured ecosystem represents a treeless oligotrophic fen with a homogeneous cover of sphagnum mosses and sedges that reach an average height of $0.25 \mathrm{~m}$ at their peak in July-August (Alekseychik et al., 2017a). The sonic anemometer Metek USA-1 mounted on a mast at a height of $3 \mathrm{~m}$ above the moss surface recorded the three velocity components $(u, v, w)$ and the sonic temperature $T_{\mathrm{S}}$ at a frequency of $10 \mathrm{~Hz}$. The instantaneous wind speed (WS) and wind direction (WD) were calculated from these measurements, as well as the mean WS and WD during each flight. The friction velocity $\left(u_{*}\right)$, the Obukhov length $\left(L_{\mathrm{O}}\right)$, and the roughness length $\left(z_{0}\right)$ were calculated using standard equations (Stull, 2011). For the purpose of reconciling the UAS spatial thermal data with the EC record, EC flux 
footprints were calculated for each flight after Kormann and Meixner (2001) using $5 \mathrm{~min}$ averages of the $10 \mathrm{~Hz}$ raw EC data (for details, see Alekseychik et al., 2017b).

\section{Results}

\subsection{Micrometeorological conditions during the flights}

The UAS thermographic retrievals were conducted around noon on two cloud-free August days in 2019, which proved to be rather different in terms of meteorological conditions. The first day (6 August) was characterized by substantial instability in terms of $z L_{\mathrm{O}}^{-1}$ and light winds, whereas $28 \mathrm{Au}-$ gust showed more near-neutral conditions and higher wind speeds (Table 2). While the stability parameter $z L_{\mathrm{O}}^{-1}$ estimated from $3 \mathrm{~m} \mathrm{EC}$ data pointed at near-neutrality on $28 \mathrm{Au}-$ gust, the higher wind speed and friction velocity indicate a predominantly mechanical or shear-induced PBL turbulence production, as opposed to 6 August when the PBL turbulence was more buoyancy produced. August is generally the time of the seasonal peak in sedge biomass that causes the annual $z_{0}$ peak (for the investigation of $z_{0}$ at this site, see Alekseychik et al., 2017a); the variation in stability explains the observation of higher $z_{0}$ on 6 August. The kinematic sensible heat flux $\overline{w^{\prime} T_{\mathrm{s}}^{\prime}}$ was slightly higher on 6 August. The mean wind speed and direction obtained by TIV are similar to the anemometric observations.

\subsection{Mean temperature field $\bar{T}(x y)$}

Figure 4 summarizes the mean temperature variations observed on the two measurement days. Because of high similarity between $\bar{T}(x y)$ of the flight pairs on both measurement days, only flight 2 of 6 August and flight 1 of 28 August are shown. In terms of mean temperature, the tree stands and open peatland form two distinct regions with the tree stands appearing overall cooler and the peatland surface warmer (Fig. 4c, f). Owing to the lack of detailed emissivity measurements, a constant emissivity of 0.98 was applied to each pixel, which might have introduced some bias in the absolute temperature values. Irrespective of the possible small biases due to the error in emissivity, the present data give a clear indication of the broad surface temperature variations (in excess of $10^{\circ} \mathrm{C}$ ) in the open peatland on the two sampling days. The overall mean TIR temperature $(\overline{\tilde{T}}(x y))$ was higher on the 6 August $\left(20.5^{\circ} \mathrm{C}\right)$ than on 28 August $\left(18.0^{\circ} \mathrm{C}\right)$, which is evident from Fig. 4 b, e. Secondly, $\bar{T}(x y)$ shows different spatial distributions. While on 28 August the highest temperatures are concentrated in a circular area in the western part of the field of view (FOV), on 6 August an additional zone of high $\bar{T}(x y)$ is observed near the northern forest edge (see the isolines in Fig. 4a, d). The peatland drainage area, part of which is formed by extensive hollow complex recognizable by its dark color in the RGB image, is characterized by the lower temperatures.

Small-scale $\bar{T}(x y)$ variability is consistent with the hollow-hummock patterning of this ecosystem. The resulting $\bar{T}(x y)$ patterning was intense on 6 August, with $17-20^{\circ} \mathrm{C}$ in the hollows and $22-25^{\circ} \mathrm{C}$ on the southern faces of the hummocks. The 28 August surface temperatures were more spatially homogeneous, probably due to stronger wind, with the hollow mean $T=18^{\circ} \mathrm{C}$ and hummock mean $T=20^{\circ} \mathrm{C}$.

The observed spatial patterns in $\bar{T}(x y)$ are not artifacts of the camera, which was assured by (i) the absence of a temporal trend in the spatial distribution of temperature maxima and (ii) the absence of significant temporal trend in the mean temperature of the frame, which, if present, would have indicated the drift due to camera stabilization and change in camera body temperature as a result of $\mathrm{WD}, \mathrm{WS}$, and $T_{\mathrm{a}}$ changes. That is to say, uncooled thermal camera measurements are always plagued by those artifacts, but in this case they were minimized and did not distort the environmental signal.

\subsection{Ground temperature fluctuations}

Figure 5 features the probability density function (PDF) of $T^{\prime}=\bar{T}(x, y)-<\bar{T}>$ observed in each of the four flights. The analysis suggests that the PDF is near-Gaussian with some minor deviations at the tails. A small, but significant, difference is in the kurtosis of the distributions, or, in other words, the maximum amplitude of temperature fluctuation. Allowing for the instrumental and processing-related noise, we may adopt the 2nd and 98th percentiles as estimates of the minimum and maximum $T^{\prime}$; on the open peatland surface, those correspond to fluctuations of \pm 0.7 and $\pm 0.6^{\circ} \mathrm{C}$ around the mean on 6 and 28 August, respectively. It is noteworthy that the surrounding coniferous forest always had a more fluctuating surface temperature that was about \pm 1.0 and $\pm 0.8^{\circ} \mathrm{C}$ on the respective days, reflecting the lower heat capacity and the higher atmospheric coupling of the conifer canopies compared to peatland surface (not shown).

The information provided in Fig. 5 is visualized spatially in Fig. 6. Not only are the excursions of ground temperature lower on the open peatland than on the other surfaces (rocky islands, tall tree stands along the south and north edges of the image), their standard deviation is similarly contrasting. The pattern in the open peatland appeared patchier on $6 \mathrm{Au}-$ gust than on 28 August. On both days, however, the standard deviation of temperature fluctuation $\left(\sigma_{T^{\prime}}\right)$ showed largescale spatial inhomogeneities: it formed faint but recognizable elongated regions of alternating low and high $\sigma_{T^{\prime}}$ extending along the mean wind direction.

The average FFT spectrum of the UAS $T_{\mathrm{g}}^{\prime}$, and sonic anemometer-derived $T_{\mathrm{s}}^{\prime}$ FFT spectrum are compared in Fig. 7. The spectra are averaged over the four flights, as the differences between the flights were minor. They exhibit the same canonical $-5 / 3$ inertial subrange slope until about $0.2 \mathrm{~Hz}$, where the UAS spectrum starts flattening for two pri- 
Table 2. Summary of the mean micrometeorological parameters determined at the EC station, where $\sigma_{\chi}$ indicates the standard deviation of an arbitrary flow variable $\chi$.

\begin{tabular}{lrrrrrrrrrr}
\hline Date, flight & $\begin{array}{r}\text { Avg period } \\
(\mathrm{min})\end{array}$ & $\begin{array}{r}\mathrm{WS} \\
\left(\mathrm{m} \mathrm{s}^{-1}\right)\end{array}$ & $\begin{array}{r}\mathrm{WD} \\
\left({ }^{\circ}\right)\end{array}$ & $\begin{array}{r}L_{\mathrm{O}} \\
(\mathrm{m})\end{array}$ & $\begin{array}{r}u_{*} \\
\left(\mathrm{~m} \mathrm{~s}^{-1}\right)\end{array}$ & $\begin{array}{r}z_{0} \\
(\mathrm{~m})\end{array}$ & $\begin{array}{r}\overline{w^{\prime} T^{\prime} \mathrm{s}} \\
\left(\mathrm{km} \mathrm{s}^{-1}\right)\end{array}$ & $\begin{array}{r}\sigma_{w} \\
\left(\mathrm{~m} \mathrm{~s}^{-1}\right)\end{array}$ & $\begin{array}{r}\sigma_{T_{\mathrm{s}}} \\
(\mathrm{K})\end{array}$ & $\begin{array}{r}z L_{\mathrm{O}}^{-1} \\
(-)\end{array}$ \\
\hline 6 August, flight 1 & 19.05 & 2.15 & 63 & -10 & 0.24 & 0.15 & 0.100 & 0.34 & 0.64 & -0.29 \\
6 August, flight 2 & 18.68 & 2.00 & 65 & -6 & 0.20 & 0.14 & 0.116 & 0.34 & 0.67 & -0.54 \\
28 August, flight 3 & 19.87 & 3.47 & 202 & -47 & 0.39 & 0.10 & 0.092 & 0.44 & 0.49 & -0.06 \\
28 August, flight 4 & 10.45 & 3.67 & 213 & -36 & 0.35 & 0.06 & 0.087 & 0.41 & 0.51 & -0.08 \\
\hline
\end{tabular}
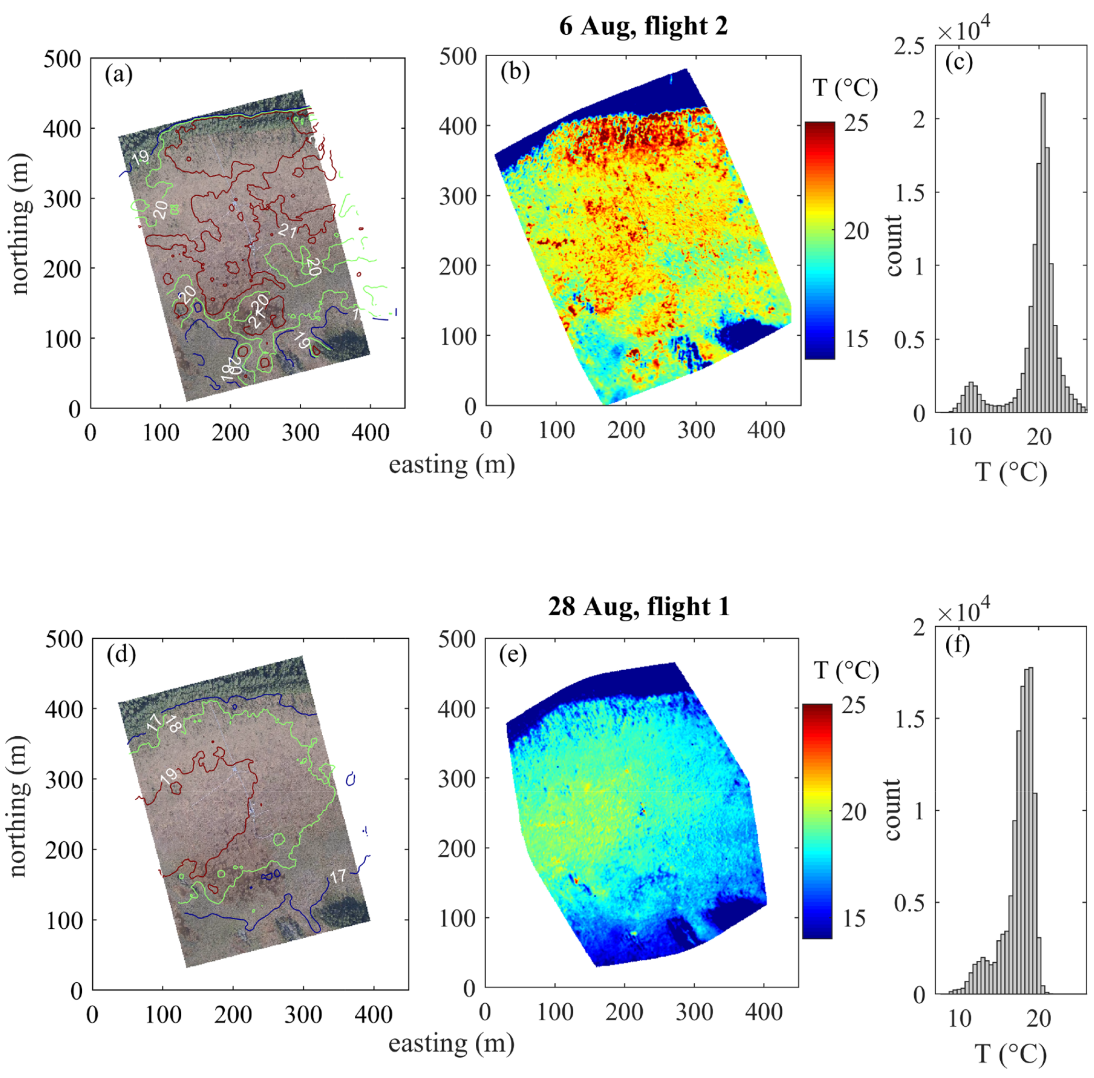

Figure 4. Pixel-mean surface temperature $(\bar{T}(x y))$ measured by UAS-mounted thermal camera for the second flight of 6 August (a-c) and first flight of 28 August (d-f). The surface emissivity was assumed uniform at 0.98. (a, d) RGB photographs with temperature isolines based on the calculated $\overline{\tilde{T}}(x y) ;(\mathbf{b}, \mathbf{e})$ surface temperature averaged for all frames of a flight $(\bar{T}(x y))$; and $(\mathbf{c}, \mathbf{f})$ histograms of $\bar{T}(x, y)$ shown in $(\mathbf{b})$ and (e).

mary reasons, namely the thermal camera noise and a high thermal inertia of the moist moss surface dampening the thermal influence of the small eddies. The -1 power law relationship (Drobinski et al., 2004; Katul et al., 1998) was not detected. The generally lower spectral energy of the UAS $T_{\mathrm{g}}$ data is due to the fact that the high thermal inertia of the ground leads to much lower surface temperature fluctuations than those observed in the airflow. The flattening of the UAS spectrum at higher frequencies results from noise contributed mainly by the thermal measurement and the image registration error.

\subsection{Detected turbulent structures and their characteristics}

In order to gain a 2D aerial view of the evolving turbulent structures, the instantaneous temperature anomaly $\left(T^{\prime}(x, y, t)\right)$ maps were combined in videos and played at a convenient rate. Distinct turbulent flow patterns were clearly visible in the $T^{\prime}(x, y, t)$ videos (see Video Supplement). Based on visual inspection, three major types of large-scale flow organization or super-structure could be distinguished (Fig. 8): (1) divergent or "fanning out" pattern, (2) quiescent period when multiple convergence and/or divergence zones 


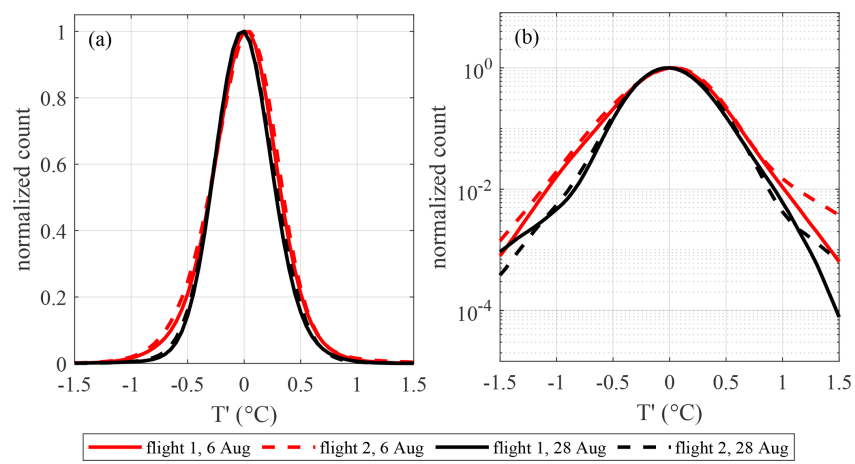

Figure 5. Normalized distributions of peatland surface temperature excursions during each flight, shown with a linear (a) and logarithmic (b) $y$-axis.

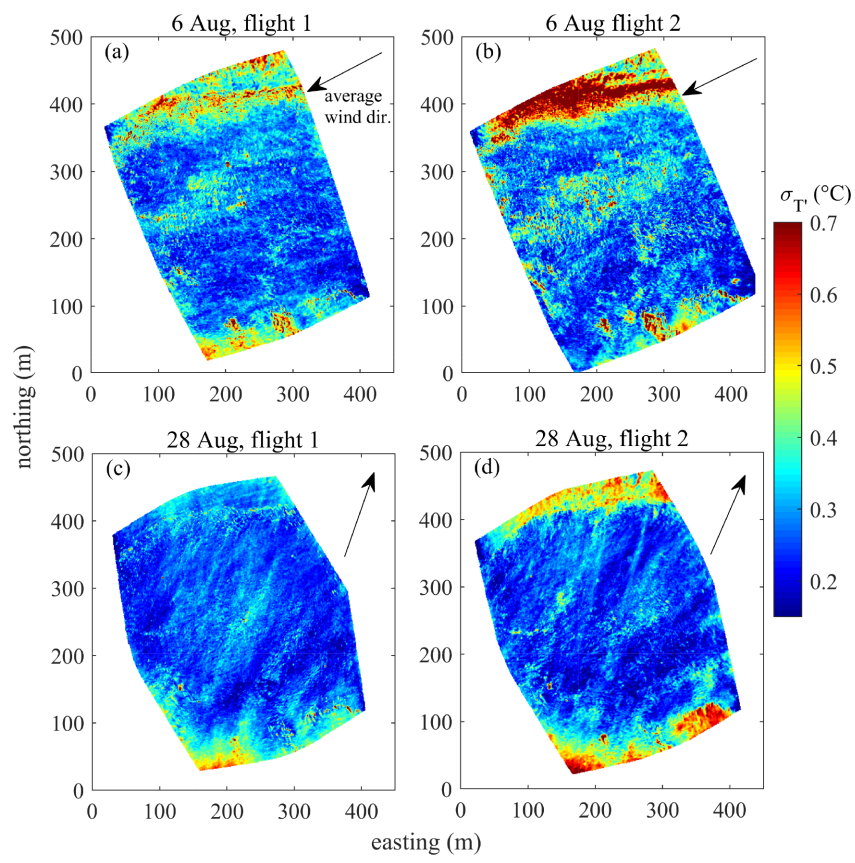

Figure 6. Maps of $\sigma_{T^{\prime}}$. The arrows show the mean wind direction during the flight.

could be observed across the FOV, and (3) elongated linear structures.

Both flights of 6 August yielded evidence of flow structure cycling between modes $1-2$ with a timescale of 2-5 min. The fanning pattern (1), when at peak strength, occupied the entire FOV and resulted in strong divergent flow pattern on the scale of several hundred meters - the wind direction is occasionally seen to differ by over $45^{\circ}$ within a single image (Fig. 8a). The "fan" consists of elongated eddies ca. 20-100 in length and $10-30 \mathrm{~m}$ in width, causing moderate ground temperature anomalies. On one occasion during the 1st flight on 6 August, the initiation of a fanning pattern is seen as an intensely cool leaf-shaped anomaly on the ground initially about $200 \mathrm{~m}$ in length with the "rays" diverg-

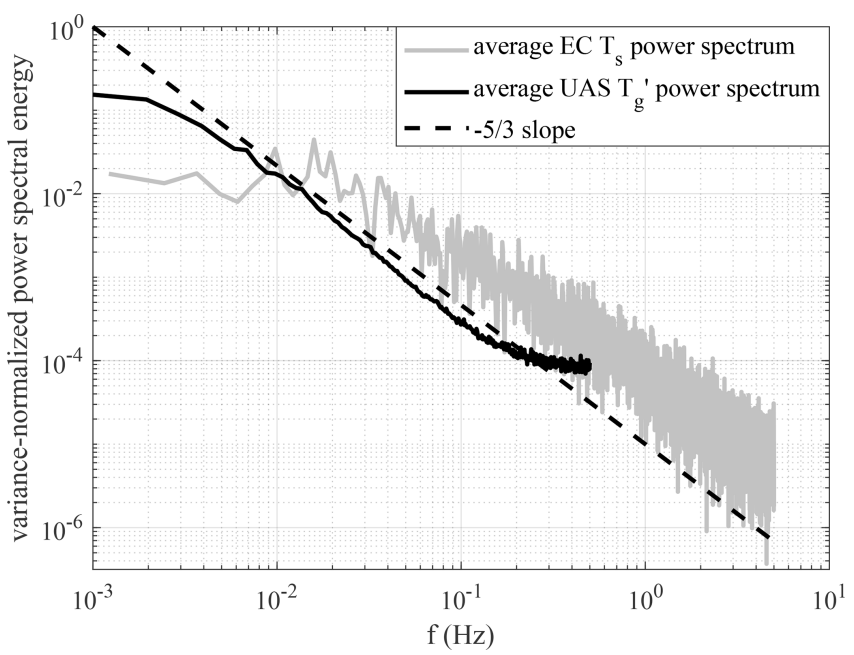

Figure 7. Normalized FFT power spectra of sonic temperature and drone temperature fluctuation. The UAS spectrum was calculated using $1 \mathrm{~Hz}$ data.
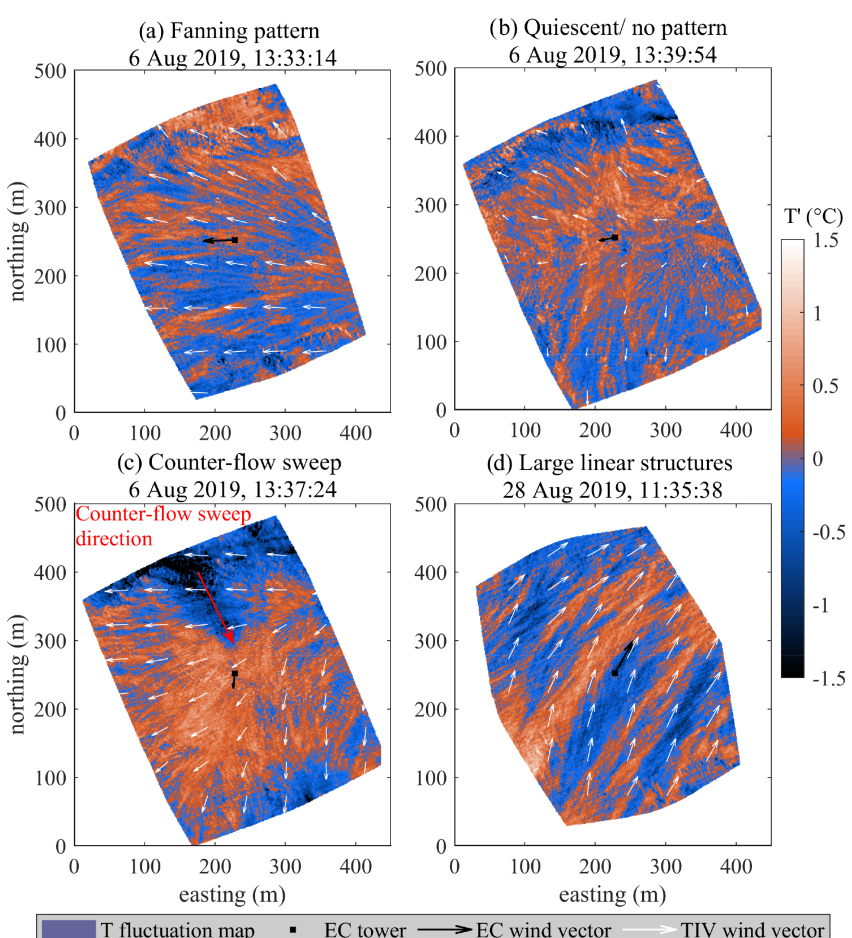

Figure 8. The typical cases of flow organization observed during the flights of 6 and 28 August 2019. The time stamps are given for the presented instantaneous $T^{\prime}$ snapshots, whereas the TIV calculations were performed using the data within $\pm 40 \mathrm{~s}$ of those times. Wind vector scale is the same for each flight, TIV, and EC; a twice longer vector is shown in (b) for ease of reading.

ing from the streamwise axis in nearly opposite directions and rapidly emanating outwards. After this initial sweep-like stage, when the ground temperature anomaly caused by the structure reaches $-1 \mathrm{~K}$, a weaker and more persistent fan- 
ning pattern as in Fig. 8a persists. The intermittent quiescent periods characterized by lower wind speed and collapse of large-scale structure are demonstrated in Fig. 8b, c. The relatively small scale of the turbulent structures $(5-50 \mathrm{~m})$ probably resulted in their being confined to the roughness sublayer and thus sensitive to large roughness changes, which may explain the well-pronounced wall effects near the forest edge (Fig. 8a, b), which was clearly shown by the contrast in temperature standard deviation (Fig. 8a, b, d).

A feature of particular interest is the onset of large sweeps seemingly dissociated from the mean near-surface flow, the most pronounced of which is displayed in Fig. 8c. The life cycle of this sweep was about 1 min from the time it reached the ground until the moment its thermal trace dissipated. Counterflow motion of somewhat less pronounced cold structures is detectable two more times during the flights of 6 August.

Contrary to 6 August, the large-scale flow on $28 \mathrm{Au}$ gust was completely dominated by pattern type 3 , persisting throughout the flight without the periods of true quiescence as was the case on 6 August. At a maximum, the length of those structures could exceed the largest dimension of the FOV (ca. $430 \mathrm{~m}$ ), their width being $30-100 \mathrm{~m}$, but judging from visual observation most structures never reached that size. The elongation of the linear structures seemed to be positively related to the periods of increased wind speed. Wall effects at the forest edge were virtually absent, implying significant vertical dimension of the impinging structures (at least substantially exceeding the roughness sublayer height above the forest stand, which is about $20 \mathrm{~m}$ tall).

The above case studies also demonstrate the success of TIV for boundary layer flow analysis. Table 3 reports average wind parameters for the illustrated cases. Here, averaging over a period of $80 \mathrm{~s}$ centered on the image timestamp was applied to both cover the interval of stationary flow required for TIV processing and account for the typical "lifetime" of large coherent structures. The average EC wind direction was within a few degrees of the average of four TIV vectors in the vicinity of the EC tower - in the cases when the flow was stationary within the FOV (Fig. 8a, b, d). In panel (c), where non-stationarity appears to be connected to the large counterflow sweep that passed through the EC sensor, the TIV WD did not average as close to the EC WD. The EC signal seems to have been dominated by that sweep, while the TIV flow field was unaffected by this temporary disturbance. Another such event is seen at the very end of the second flight of 6 August (see the corresponding video). In terms of wind speed, there is a difference between spatially homogeneous and stationary flow field (a, d) and inhomogeneous and/or non-stationary flow $(b, c)$ : in the former, the EC WS was higher; in the latter, the two estimates were similar.

The periodicity of the turbulent motions was further investigated by analyzing the continuous $2 \mathrm{D}$ wavelet transforms on the spatial scales of $1-50 \mathrm{~m}$. The result is presented in the form of scalograms normalized by the means of the spec-
Table 3. Mean wind parameters for the cases in Fig. 8.

\begin{tabular}{lrrrr}
\hline & $\begin{array}{r}\text { TIV } \bar{U} \\
\left(\mathrm{~m} \mathrm{~s}^{-1}\right)\end{array}$ & $\begin{array}{r}\text { EC } \bar{U} \\
\left(\mathrm{~m} \mathrm{~s}^{-1}\right)\end{array}$ & $\begin{array}{r}\text { TIV WD } \\
\left({ }^{\circ}\right)\end{array}$ & $\begin{array}{r}\text { EC WD } \\
\left({ }^{\circ}\right)\end{array}$ \\
\hline Fig. 8a & 1.8 & 2.9 & 100 & 88 \\
Fig. 8b & 0.8 & 0.7 & 82 & 78 \\
Fig. 8c & 1.7 & 1.4 & 41 & 6 \\
Fig. 8d & 2.4 & 3.5 & 201 & 208 \\
\hline
\end{tabular}

tral density at the respective scales in Fig. 9. From Fig. 9, it becomes evident that periods of intensified turbulence were more frequent on 28 August (flights 3-4) than on 6 August (flights 1-2). The normalized power generally varies more at the large spatial scales than on the small scales. A conspicuous feature is contributed by the periods of strong wavelet power increase across the larger scales, waning somewhat in the lower scales. By comparison with the $T^{\prime}$ videos, one finds that they correspond to the events of strong flow, which took the form of fanning events during flights 1 and 2 . The strong fanning event in the beginning of flight 2 left a particularly sharp signature (Fig. 9b). The waves of elongated parallel structures during flights 3 and 4 left a similar signature, but had shorter time spans in accordance with their lifespan. Another interesting signature also emerges: an isolated region of increased power that only encompasses a narrow range of scales. Three such "scale-dependent" bursts may be seen during flight 3 around $t=[400,650,750] \mathrm{s}$ (Fig. 9c). At the same time, the periods of low wavelet power represent the "quiescent" conditions when well-defined large-scale structures were absent, such as that illustrated in Fig. 8b.

The spectral properties of the ground temperature fluctuations were studied by dividing the signal into their alongand cross-wind spectra. Now, each $T^{\prime}(x, y, t)$ image was rotated and interpolated on a rectangular grid to direct the mean wind along the $x$-axis, with the $y$-axis being a cross-wind coordinate. The wind direction used to perform this rotation was calculated as the average anemometer WD for the period of $\pm 30 \mathrm{~s}$ around the timestamp of an image. FFT power spectra were calculated for rows and columns of the rotated images on the scales of 2-128 $\mathrm{m}$ and averaged, yielding the mean along- and cross-wind spatial power spectra. Rows and columns left with less than 300 pixels $(=300 \mathrm{~m})$ after rotation were excluded as unrepresentative of the largest spatial scales.

Two metrics are used to describe the relations between the fluctuations on different spatial scales and the along and cross wind directions, namely, (i) the ratio between the spectral powers at 128 and $10 \mathrm{~m}$ for both along- and cross-wind directions, and (ii) the ratio between the along-wind power at $128 \mathrm{~m}$ to cross-wind power at $128 \mathrm{~m}$. Metric (i) can be interpreted as a measure of domination of large coherent structures at a given time and also as a measure of anisotropy when the ratios for along- and cross-wind directions are com- 

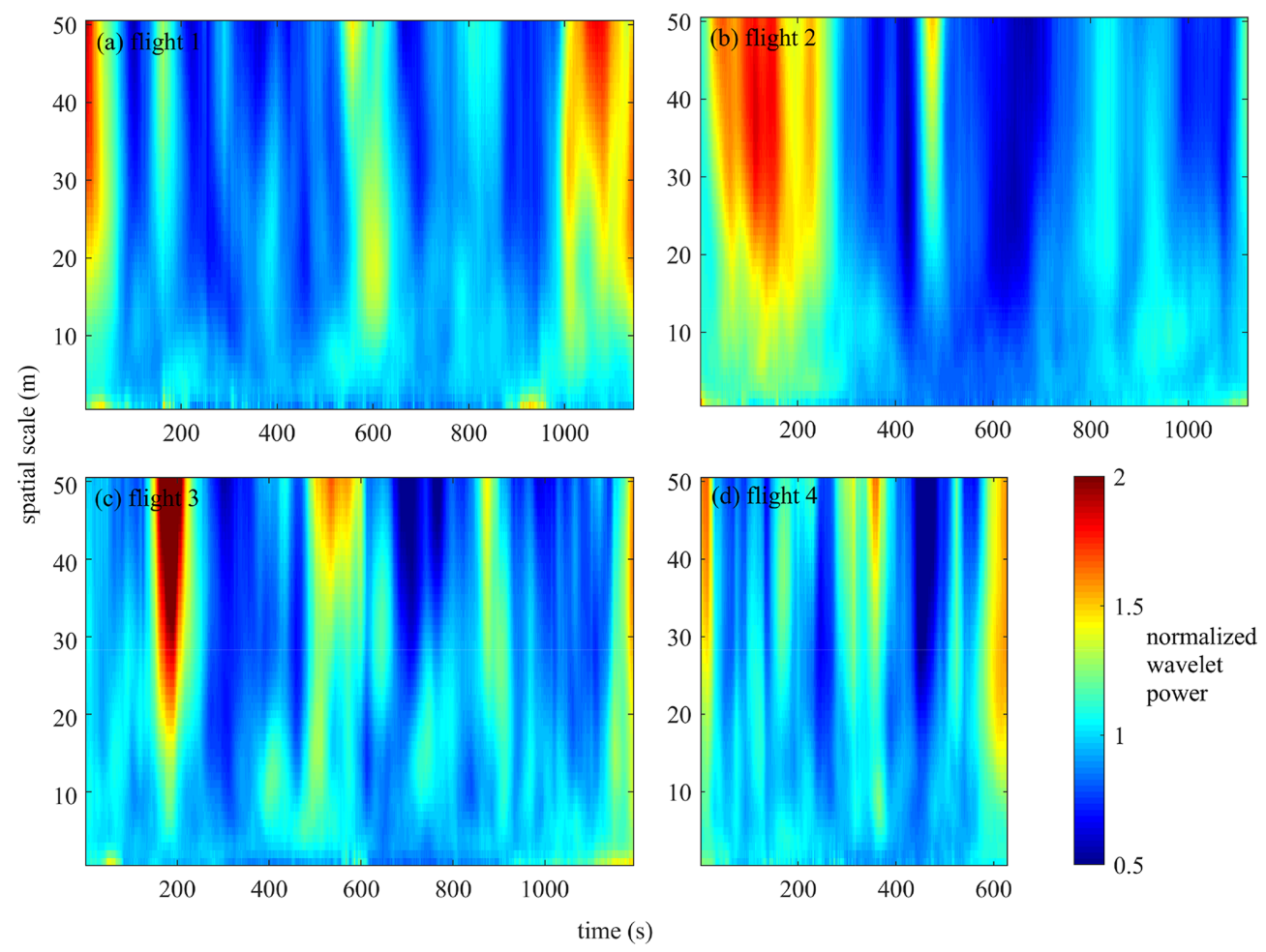

Figure 9. Scalograms of 2D continuous wavelet transforms on the spatial scales of $1-50 \mathrm{~m}$. Absolute values of wavelet power are taken in order to average over the negative and positive $T$ excursions and normalized by the average absolute power at the respective scale.

pared. While (ii) is a measure of anisotropy, it is directly related to the largest captured scale of $128 \mathrm{~m}$. The two metrics are plotted in Fig. 10. It should be noted that the $128 \mathrm{~m}$-scale structures have a characteristic length scale of $\sim 10^{-2} \mathrm{~Hz}$, i.e., correspond to the energy containing subrange (Fig. 7). Consequently, the smaller eddies (under ca. $100 \mathrm{~m}$ in size) fall in the inertial subrange.

Most obvious is the striking dissociation between the along-wind and cross-wind ratios of the $128 / 10 \mathrm{~m}$ spectral power metric (i) and their intense individual variability. Generally, for both directions, the periods of increased ratios (i) correspond to the periods of intensified ground temperature fluctuations highlighted in Fig. 9, while the opposite is true for the quiescent periods as seen in Fig. 9. The tallest peaks reach the value of about 80 , indicating the total dominance of large-scale coherent structures over smaller-scale nearground turbulence. Most of the time, the ratios for the alongand cross-wind directions are anti-correlated in the sense that typically only one of the two may peak at any given time. For example, the pronounced coherent events detected earlier at the end of flight 1 , beginning of flight 2 , and during flight 3 are associated with a peaking $128 / 10 \mathrm{~m}$ power ratio, indicating the contact of large coherent structures with the ground at those times. However, during flight 3 the highest values are attained by the along-wind $128 / 10 \mathrm{~m}$ power ratio, in contrast to the first two flights where the cross-wind ratio was higher.
The ratio of the $128 \mathrm{~m}$ spectral powers metric (ii) attains values of $0.2-5$ and displays different dynamics during flights $1-2$ and 3 , being generally below unity in the former case and more often above unity in the latter case. It should be noted that the periods of increased metric (i) for cross-wind direction generally correspond to the troughs in metric (ii), as both require the cross-wind spectral power at $128 \mathrm{~m}$ scale to be high.

\subsubsection{Eddy size and shape derived from 2D wavelet transforms}

The eddy sizes and shapes extracted from the 2D wavelet transform are shown in Fig. 11. The algorithm (Sect. 2.2.6 and Fig. 3) detected typically 5-20 eddies per image, the eddy sizes varying between $70-240 \mathrm{~m}$ in length and $20-80 \mathrm{~m}$ in width and having areas of $1000-8000 \mathrm{~m}^{2}$ (see Fig. 3d for an example of the derived eddy parameters). The eddy parameter distributions are roughly Gaussian and display a modest but significant progression of the median eddy dimensions and area in the flight order 2-1-4-3. Flights 1-2 are similar, flight 4 being moderately different from them, whereas flight 3 stands separate from the other three flights. In flight 3, the detected major axes were longer while the minor axes were shorter, providing for the highest eddy length/width ratio of all flights. The eddy areas observed during that flight were generally the largest as well. 

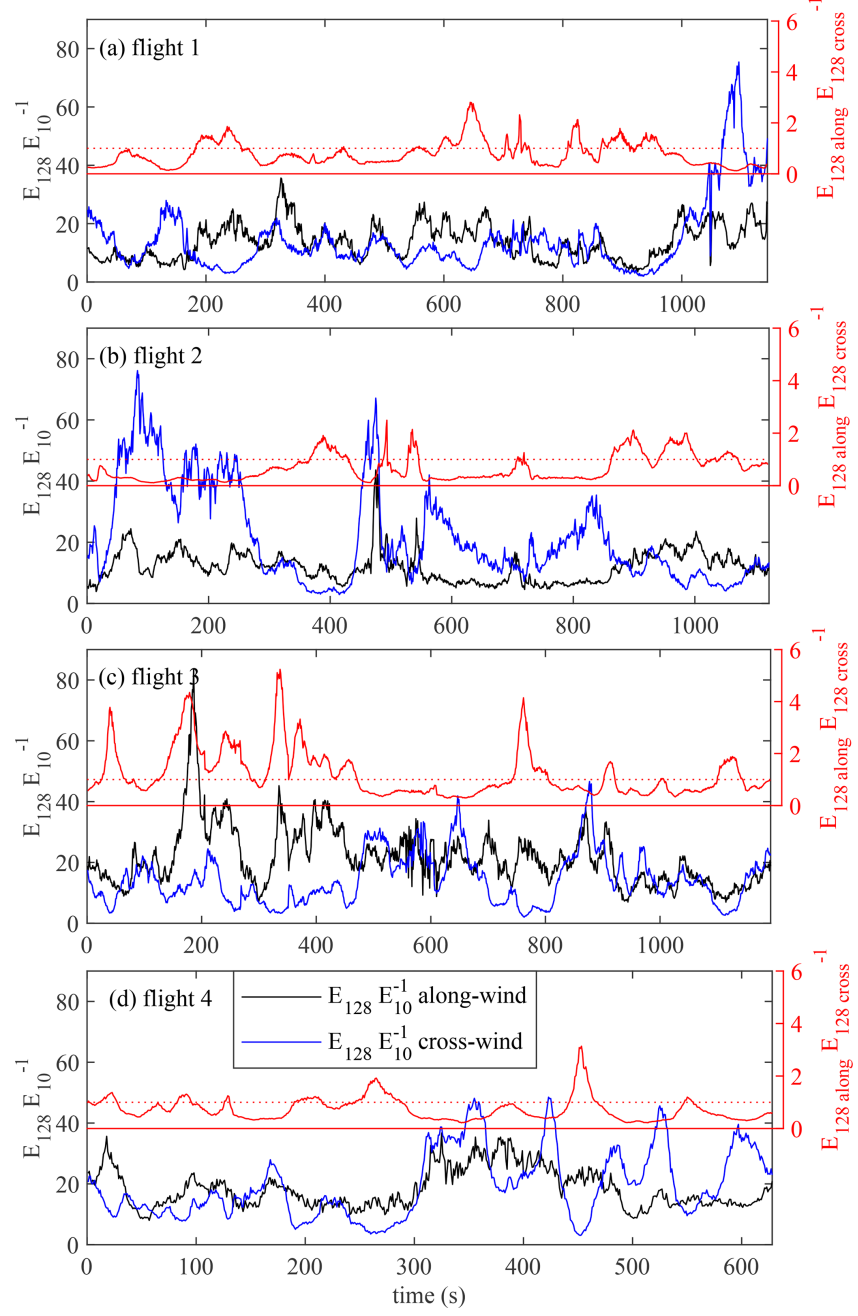

Figure 10. The ratio between the FFT spectral powers at the scales of 128 to $10 \mathrm{~m}$ for the along- and cross-wind directions (black and blue lines, respectively) and the along- to cross-wind spectral power ratio at the scale of $128 \mathrm{~m}$ (red lines).

The orientation of the eddies also varied in time in close agreement with the wind direction averaged over 1 min interval centered on the thermogram record time (not shown). The thermogram-derived $14 \mathrm{~m}$ scale eddy orientations, however, show a systematic, variable $0-20^{\circ}$ clockwise rotation relative to the anemometer wind in each of the four flights. The elongation of eddies was, therefore, collinear with the wind direction, but the directional difference increased during the quiescent periods due to the difficulty of determining the orientation of the more circular eddies that then dominated and the wide range of eddy orientations detected within a single image.

The relationship between the 5 min average eddy properties (size and shape), diabatic stability $\left(z L_{\mathrm{O}}^{-1}\right)$, and $u_{*}$ is shown in Fig. 12a-d. Eddy length-to-width ratio is in positive correlation with both quantities (Fig. 12a-b), implying the residence of more elongated coherent structures during the periods of lower instability and intensified mixing. The relationship between eddy area, $z L_{\mathrm{O}}^{-1}$, and $u_{*}(\mathrm{Fig} .11 \mathrm{c}-\mathrm{d})$ is less strong. Eddy length/width ratio is also positively correlated with eddy area (Fig. 12e) meaning that large eddies are typically elongated along the mean wind. The data in Fig. 12 are shown partitioned into warm and cool eddies to examine the possible differences; the offsets between the eddies of different signs reach $10 \%-15 \%$ in relative expression but fail to form a clear trend against $u_{*}$ or stability.

\subsubsection{Comparison of air and ground temperature excursions}

The drone and EC-derived quantities $\left(\sigma_{T_{\mathrm{g}}}, \sigma_{T_{\mathrm{s}}}\right.$, and mean $T^{\prime}$ difference between the positive and negative eddy regions $\left.\Delta\left(T_{\mathrm{g}}^{-}, T_{\mathrm{g}}^{+}\right)\right)$were again averaged over $5 \mathrm{~min}$ periods to achieve a finer temporal resolution roughly matching the timescale of the coherent structures. First, we note that temperature fluctuations measured in the air and on the ground are clearly correlated (Fig. 13a-c); the statistics of ground temperature fluctuations show $R^{2}$ of $0.33-0.38$ against the standard deviation of sonic temperature. However, when removing "outliers" marked with * and \# in (b) and (c), the $R^{2}$ increases up to 0.6. Upon checking with the previously identified chronology of the coherent structure events, we find that those 5 min periods appearing to be outliers were contemporaneous with the passage of major cool coherent structures in the beginning of flights 2 and 3 (see Fig. 9). In comparison, the regressions of the same quantities against the kinematic heat flux are considerably more scattered (Fig. 13d-f).

\section{Discussion}

A UAS comprised of quadcopter DJI Matrice $210 \mathrm{~V} 2$ and camera DJI Zenmuse XT2 was capable of hovering at the altitude of $500 \mathrm{~m}$ for a maximum time of $20 \mathrm{~min}$, while continuously recording surface temperature pointing at nadir. The setup was beneficial for the measurement of impingement of PBL turbulence on the surface. On one hand, the record length approaches the typical averaging period of the groundbased eddy-covariance data, and was sufficient to analyze the periodicity of the large coherent turbulent structures forming in a summertime convective PBL over a peatland. On the other hand, the size of an area seen from $500 \mathrm{~m}$ height with a $13 \mathrm{~mm}$ lens was $430 \times 340 \mathrm{~m}$, which enables imaging coherent turbulent structures of considerable size. With these dimensions, the requirement that the FOV should be bigger than the integral length scale of turbulence, proposed by Christen et al. (2012), is met. A nadir direction of view also resulted in a uniform thermal resolution at the surface of about $0.6 \mathrm{~m}$ per pix, which was high enough to resolve the smaller eddies down to the sizes of 1-2 m. Therefore, 

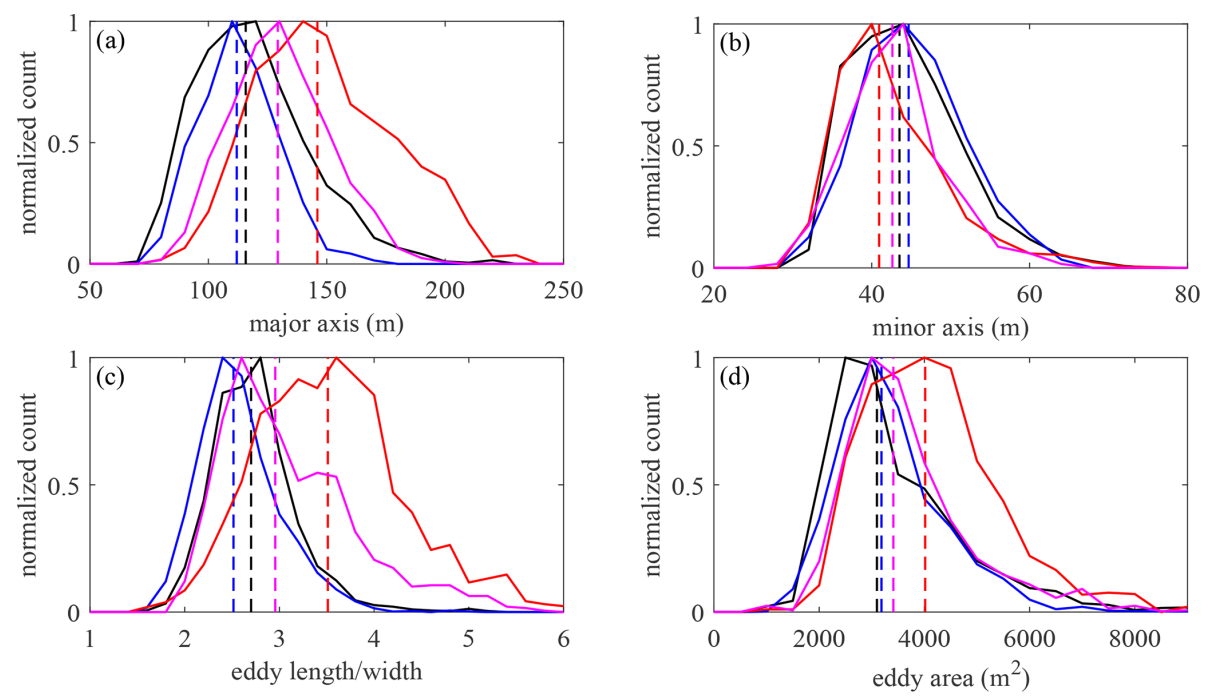

Flight $1-$ Flight $2-$ Flight $3-$ Flight 4

Figure 11. Distributions of size parameters of the eddy thermal traces derived from the 2D wavelet transforms. The vertical dashed lines mark the distribution medians. The major axis is the greater dimension of the coherent structure's thermal trace ("length"), which is always oriented in streamwise direction; correspondingly, the minor axis is the "width".
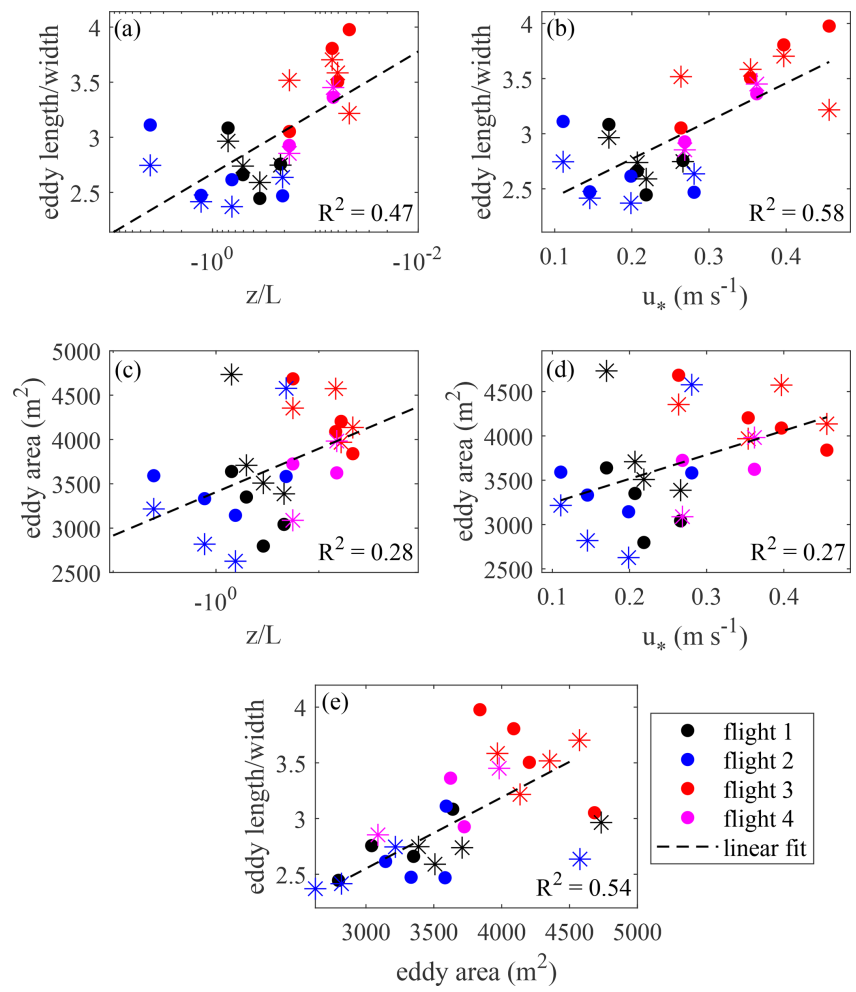

Figure 12. (a-d) Relationship between the 5 min average properties of eddy thermal traces (mean area, length-to-width ratio) and the corresponding averages of $z / L_{\mathrm{O}}$ and $u_{*}$; (e) length-to-width ratio versus mean area. The $*$ indicates eddy traces with positive mean $T^{\prime} ; \bullet$ indicates those with negative mean $T^{\prime}$. the present TIR study has a number of advantages over the prior work in four ways: (a) the surveyed area is the largest; (b) the camera was aimed at nadir, minimizing the geometric distortion across the thermogram; (c) the $30 \mathrm{~Hz}$ record rate, even when downsampled to $1 \mathrm{~Hz}$, is sufficient to resolve some of the inertial-scale turbulent eddies; and (d) the UAS platform can be positioned at an arbitrary point in space and thus, e.g., produce imagery overlapping with the footprint of the other measurements (such as the eddy covariance in this case). Thus, this is the first successful attempt to use a drone to explore a wide spectrum of eddy sizes impinging on the surface via TIR imaging.

The current image analysis approach to identify turbulent coherent structures has a significant advantage over analyzing fixed sensor data on atmospheric state variables ("1D approach" in the following) in that it provides 2D images of coherent structures that are readily recognizable with the naked eye or by automated algorithms. A problem inherent to the traditional fixed-sensor meteorological observation is that the center of a coherent structure might pass at any distance from the sensor, thus making the "slice" observed in the recorded time series an unreliable representation of temperature fluctuation caused by the actual structure. In contrast, 2D imaging allows for identification of the turbulent structure shape (from its fingerprint on the ground) and its position relative to ground at any moment in time. However, even experiments employing 2D thermal imagery tended, in the past (Christen et al., 2012; Garai and Kleissl, 2011; Inagaki et al., 2013), to drift towards a "statistical" perception of the coherent structures, forgoing all the potential to get a hold of the actual geometry and movement of the individual structures offered 

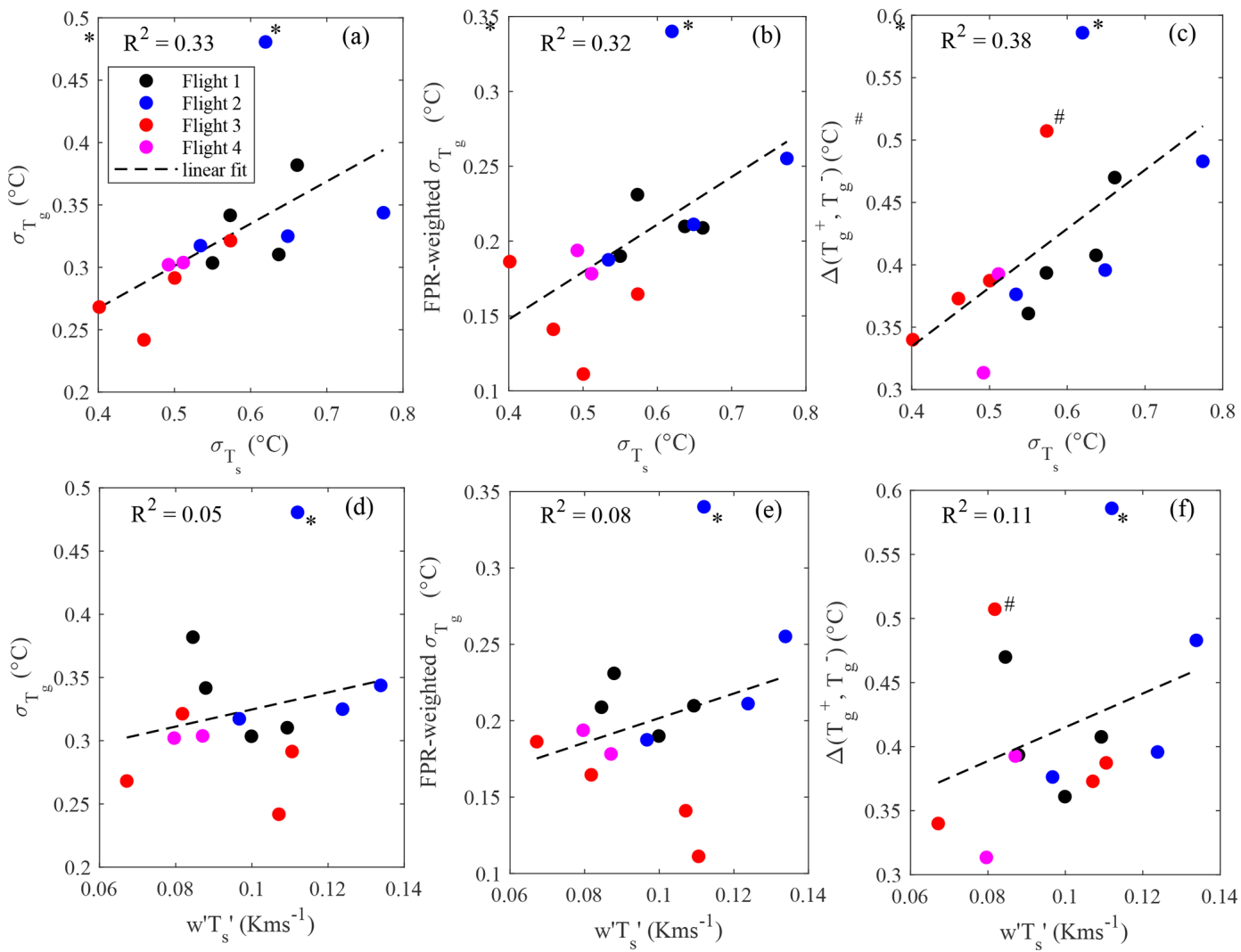

Figure 13. Drone data (a - standard deviation of the ground temperature; $\mathbf{b}$ - EC footprint-weighted standard deviation of the ground temperature, footprint based on Kormann and Meixner, 2001; $\mathbf{c}$ - difference between the mean temperature excursions in cold and warm eddy traces) against the standard deviation of sonic temperature. In (d)-(f), the same quantities as in (a)-(c) are plotted against the kinematic sensible heat flux. * and \# indicate the outlying 5 min periods in the beginnings of flights 2 and 3 when particularly strong coherent structures occurred.

by 2D TIR. Furthermore, a 1D approach relies on turbulence being ergodic, as a result, precluding the segregation of nonstationary events from the mean flow, such as the counterflow event in Fig. 8c; such non-stationarities have not been addressed in the previous studies either. This study attempted to avoid these pitfalls by focusing on spatiotemporal analyses.

The assumption underlying our approach to the imaging of turbulence is that the coherent structures dominating the surface layer flow, such as thermals, hairpins, and roll vortices remain attached to the ground for a period long enough for their evolution to be described, and this attachment is sufficiently "tight" to provide information on their internal structure. This assumption finds support in the similarity of the structures observed in this UAS experiment to those found earlier in many studies employing DNS (Fang and PortéAgel, 2015; Laima et al., 2020), Doppler radar (Newsom et al., 2008), TIV (Inagaki et al., 2013), and other methods.

The 2D organization of PBL turbulence was very disparate on the two field days. Given the difference in meteorological conditions and micrometeorological parameters, one might expect some difference in the organization of turbulence and much evidence has emerged in this study to support this view. It appears that the conditions of 28 August, characterized by smaller instability but more intensive mixing due to stronger wind on 6 August (Table 2), led to the formation of larger and more elongated coherent structures (Figs. 8, 11). Contrastingly, when the wind picked up on the 6 August, the dominant large-scale structure was a field of smaller eddies diverging in a fan-shaped manner. The mixing on 28 August was apparently contributed by shear stress and mechanical turbulence, whereas on 6 August it was rather controlled by buoyancy and convection, which we think is the primary reason for such a substantial difference in the large-scale PBL turbulence organization. The same driver is perhaps responsible for the slower development and longer survival of selfsustaining large-scale turbulent structures on the 6 August. This result is in line with the LES study of Margairaz et al. (2020) who observed flow organization regimes depending on the magnitude of geostrophic forcing and buoyancy. There is also qualitative agreement with the proposed dependency of the size and elongation of the dominant momentumtransporting eddies on stability (Salesky et al., 2013). 
The coherent structures were aligned with the wind direction, which was shown independently by comparison between the anemometric data with flow directions obtained with the help of TIV (Fig. 8, Table 3) and the orientation of eddies detected by the 2D wavelet-based algorithm (Fig. 11). However, the TIV algorithm provided for a closer match with the EC wind by virtue of its relying on the small-scale turbulence, whereas the larger-scale structures whose orientation was calculated explicitly proved to deviate by $20^{\circ}$ clockwise and did not follow the EC wind trend on the short timescale. Differences between the coherent structure translation direction and the EC WD were also noted in earlier works (e.g., Wilczak and Tillman, 1980). The advection speed of the small-scale turbulence determined by TIV was also close to the EC wind speed.

The differences in periodicity and self-organization of turbulence on the two measurement days were assessed by spatial spectra in two ways: by 2D wavelet transform and crossversus along-wind FFT spectra. The 2D wavelet scalograms (Fig. 9) show transitions from high to low spectral power that are associated with impingement and dissipation of large coherent structures, which happened more frequently on the 28 August than 6 August. The two metrics constructed of cross- and along-wind FFT spectra (Fig. 10) support the periodicity and give a general indication that the periods of increased power in Fig. 10 were the times when large structures elongated along WD dominated.

The present study paves the way to the determination of surface sensible heat flux based on UAS thermal videos. Firstly, there are indications that the flux variance technique (Albertson et al., 1995) may be adapted to the calculation of sensible heat flux from UAS thermal videos. As shown in Fig. 13a-c, the correlation between the standard deviations of sonic temperature and UAS temperature may be sufficiently high to parameterize $\sigma_{T_{\mathrm{s}}}$ as a function of $\sigma_{T_{\mathrm{g}}}$ and use it in the flux variance expression of Albertson et al. (1995). The data set of the present study is, however, too short to consistently verify the validity of the approach. Care should be exercised in interpreting the UAS-derived heat fluxes, as the UAS-derived quantities that are expected to be linked to surface heat flux show a lot of scatter against EC kinematic heat flux $\left(w^{\prime} T^{\prime}\right.$, Fig. 13d-f). This may be due to a variety of reasons, e.g., the invalidity of the footprint model calculated with $5 \mathrm{~min}$ averages or the lack of direct link between $\sigma_{T^{\prime}}$ and $w^{\prime} T^{\prime}$ on a $5 \mathrm{~min}$ timescale. However, we propose that if the eddies are large, attached to the ground, contribute most to heat transport, and the flow is ergodic, space-time mean $\sigma_{T_{\mathrm{g}}}$ of the eddy at the ground can ultimately be used to derive heat flux caused by the impingement of an eddy. Alternatively, a "pixel" type flux variance can be used to infer variations in sensible heat flux, where heat flux is calculated for individual pixels using their specific $\sigma_{T_{\mathrm{g}}}$. Secondly, the possibility to segregate large eddies and derive the durations of their contact with the ground enables the use of a modified surface renewal approach (Paw U et al., 1995), in which the amplitude of temperature excursion and period of an eddy are the drivers of heat flux. Calculating the instantaneous soil heat flux as a function of ground surface temperature and deriving sensible heat flux as a residual of the energy budget results in the third method for sensible heat flux calculation based on TIR imaging (Morrison et al., 2017), although in non-arid ecosystems it would be strongly dependent on the observations of both net radiation and latent heat flux.

\section{Conclusions}

The present study develops a framework for planetary boundary layer turbulence analysis based on UAS thermal camera measurements. The methods for thermal sequence retrieval, its post-processing, and detection of large coherent structures were proposed. The performance and validity of the methods were tested in a case study over a flat and treeless boreal peatland in southern Finland. The spectral and morphological analysis pointed at the domination of large coherent structures up to tens of meters in width and hundreds in length, as expected in a convective PBL. Wind parameters independently observed by ground-based eddy-covariance setup provided support to the turbulence statistics derived by thermal sequence analysis. However, the novel 2D approach of this study also allowed for detection of instationary events such as counterflow sweeps, which is beyond the capacity of previous observational methods. Larger, longer, and more linear eddies were associated with lower instability as expressed by the stability parameter $z L_{\mathrm{O}}^{-1}$, while smaller and more circular eddies were observed at higher instability. The association between the surface temperature fluctuations on the ground and in the air, and the possibility to directly infer the residence time of an eddy and $T$ fluctuation created by it, prepare ground for the application of sensible heat flux estimation by flux variance (Albertson et al., 1995) and surface renewal (Paw U et al., 1995) methods. 


\section{Appendix A: Details of the vignetting correction}

The fourth-order polynomial describing the vignetting effect (see Fig. A1) is described by

$$
\begin{aligned}
S(x, y) & =p_{0,0}+p_{1,0} \cdot x+p_{0,1} \cdot y+p_{2,0} \cdot x^{2} \\
& +p_{1,1} \cdot x \cdot y+p_{0,2} \cdot y^{2}+p_{3,0} \cdot x^{3} \\
& +p_{2,1} \cdot x^{2} \cdot y+p_{1,2} \cdot x \cdot y^{2}+p_{0,3} \cdot y^{3} \\
& +p_{4,0} \cdot x^{4}+p_{3,1} \cdot x^{3} \cdot y+p_{2,2} \cdot x^{2} \cdot y^{2} \\
& +p_{1,3} \cdot x \cdot y^{3}+p_{0,4} \cdot y^{4},
\end{aligned}
$$

with $x$ and $y$ being the $x$ - and $y$-coordinate of an image pixel and $p_{i, j}$ the fit parameter value. The derived lens-specific coefficient values are given in Table A1.

The vignetting correction matrix was obtained by subtracting the polynomial fit from the mean temperature of the center. We assumed that vignetting is zero at the center and computed the mean temperature from of the $40 \times 40$ pixel square zone at the center of the image. Finally, vignetting was eliminated by adding the correction matrix to each frame obtained in the field.
Table A1. Coefficients of the fourth-order polynomial surface describing the vignetting effect.

\begin{tabular}{lrr}
\hline Coefficient & Value & $95 \%$ CI \\
\hline$p_{0,0}$ & 23.79 & $23.78,23.79$ \\
$p_{1,0}$ & 0.02014 & $0.0201,0.02019$ \\
$p_{0,1}$ & 0.01298 & $0.01292,0.01303$ \\
$p_{2,0}$ & $-5.922 \times 10^{-5}$ & $-5.944 \times 10^{-5},-5.9 \times 10^{-5}$ \\
$p_{1,1}$ & $-5.36 \times 10^{-5}$ & $-5.382 \times 10^{-5},-5.338 \times 10^{-5}$ \\
$p_{0,2}$ & $-3.069 \times 10^{-5}$ & $-3.104 \times 10^{-5},-3.034 \times 10^{-5}$ \\
$p_{3,0}$ & $8.734 \times 10^{-8}$ & $8.687 \times 10^{-8}, 8.782 \times 10^{-8}$ \\
$p_{2,1}$ & $8.291 \times 10^{-8}$ & $8.245 \times 10^{-8}, 8.336 \times 10^{-8}$ \\
$p_{1,2}$ & $9.391 \times 10^{-8}$ & $9.334 \times 10^{-8}, 9.449 \times 10^{-8}$ \\
$p_{0,3}$ & $2.667 \times 10^{-8}$ & $2.574 \times 10^{-8}, 2.761 \times 10^{-8}$ \\
$p_{4,0}$ & $-6.603 \times 10^{-11}$ & $-6.639 \times 10^{-11},-6.567 \times 10^{-11}$ \\
$p_{3,1}$ & $-3.4 \times 10^{-12}$ & $-3.795 \times 10^{-12},-3.006 \times 10^{-12}$ \\
$p_{2,2}$ & $-1.465 \times 10^{-10}$ & $-1.47 \times 10^{-10},-1.46 \times 10^{-10}$ \\
$p_{1,3}$ & $4.615 \times 10^{-13}$ & $-1.547 \times 10^{-13}, 1.078 \times 10^{-12}$ \\
$p_{0,4}$ & $-2.202 \times 10^{-11}$ & $-2.29 \times 10^{-11},-2.114 \times 10^{-11}$ \\
\hline
\end{tabular}

Original thermogram and fit

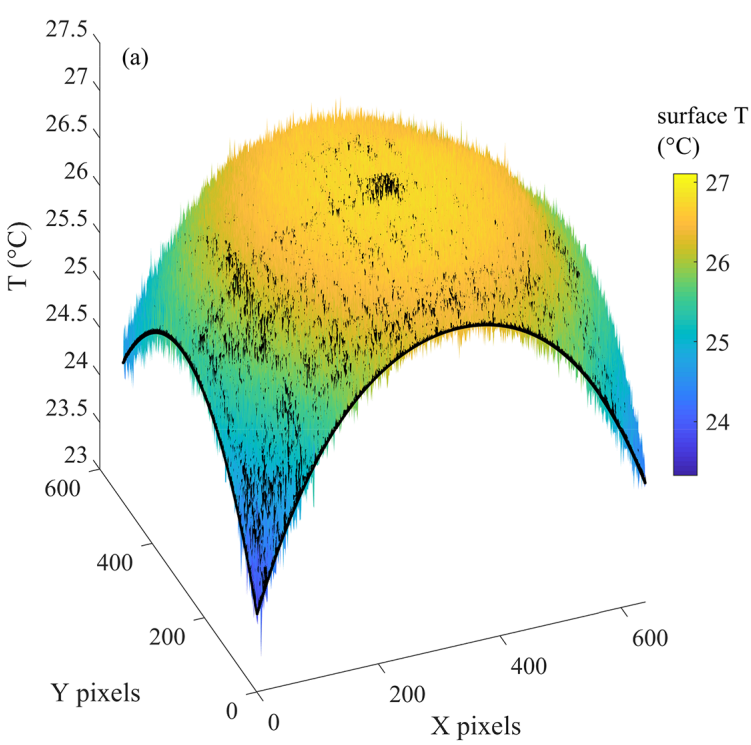

(b)

Original thermogram
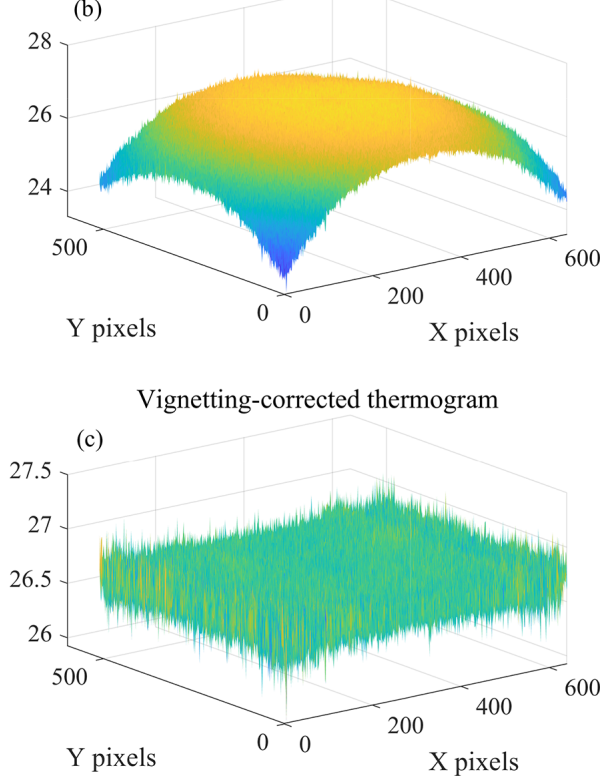

Figure A1. (a) Fourth-order polynomial fit (black surface) and the thermogram of the black fabric (RMSE $=0.08 \mathrm{~K})$; (b) uncorrected thermogram of black fabric surface (same data as in a); (c) vignetting-corrected thermogram. 


\section{Appendix B: Camera intrinsics}

The geometric calibration of the TIR camera was performed using a checkerboard so as to obtain the camera intrinsic parameters given in Table B1. Figure B1 gives a screenshot of the geometric calibration process in the MATLAB ${ }^{\circledR}$ camera calibrator tool.

Table B1. Camera intrinsic parameters.

\begin{tabular}{lcc}
\hline Parameter & \\
\hline Radial distortion & $k_{1}=-0.049$ & $k_{2}=0.646$ \\
Tangential distortion & $p_{1}=0.0035$ & $p_{2}=0.0028$ \\
Focal length & $f_{x}=f_{y}=801 \mathrm{~mm}$ \\
Principal point & $c_{x}=327$ pix $\quad c_{y}=276$ pix \\
Skew & $\theta=3.4796^{\circ}$ \\
Mean reprojection error & 0.26 pixels \\
\hline
\end{tabular}
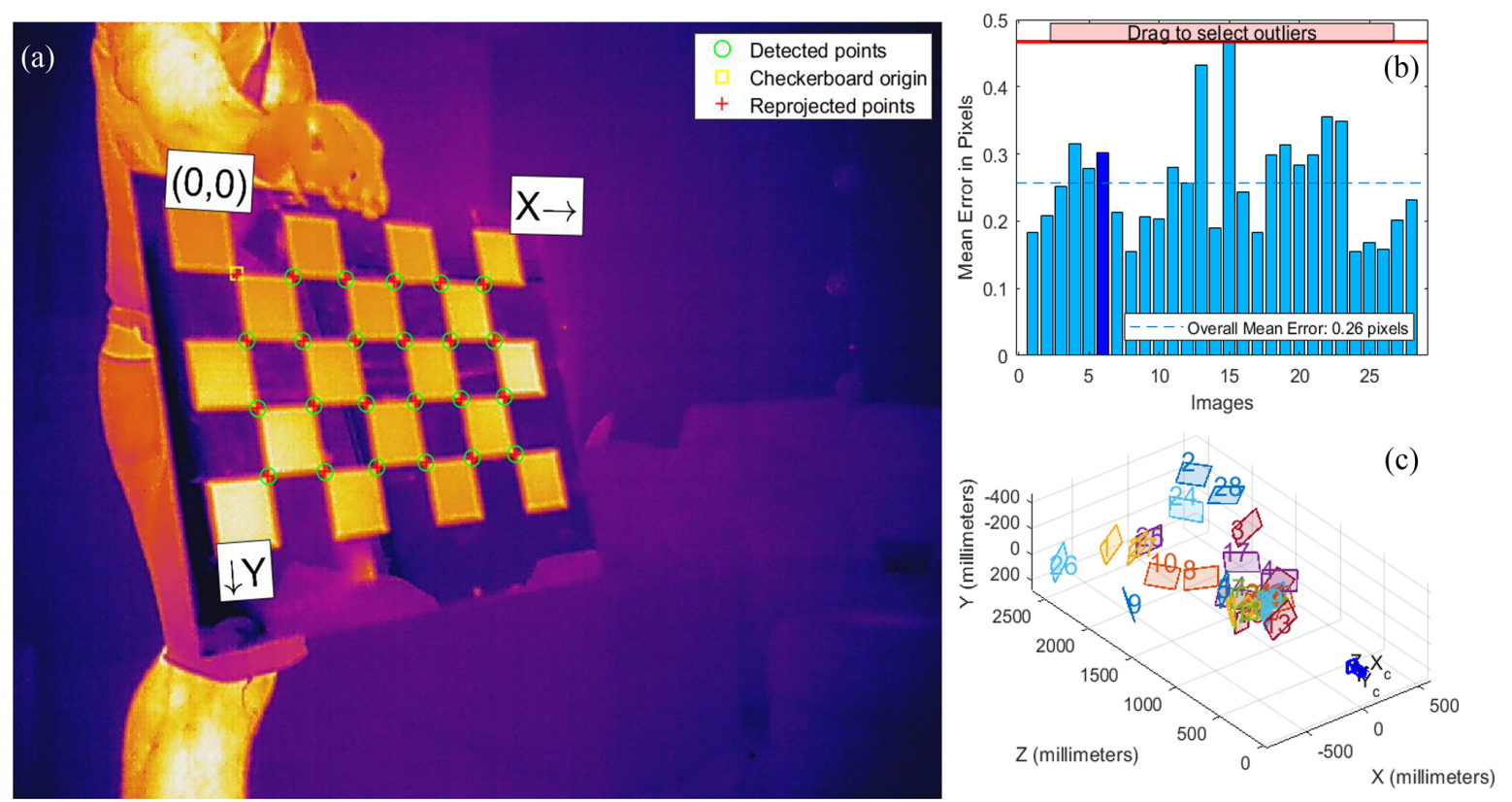

Figure B1. Screenshot from the MATLAB ${ }^{\circledR}$ camera calibrator tool. (a) One of the 28 images used to derive the calibration parameters; (b) mean reprojection error (pix) for each image; (c) relative positions of the checkerboard relative to the camera. 


\section{Appendix C: Image similarity metrics}

Structural Similarity Index (SSIM) is a measure of the similarity between two images that simultaneously uses mean pixel values, pixel standard deviations, and pixel cross correlations in order to assess the differences in luminance, contrast, and image structure, respectively (Renieblas et al., 2017):

$\operatorname{SSIM}=\frac{1}{X Y} \sum_{x=1}^{X} \sum_{y=1}^{Y}[l(x, y)]^{\alpha} \cdot[c(x, y)]^{\beta} \cdot[f(x, y)]^{\gamma}$,

where $X$ is the number of pixels in horizontal dimension, $Y$ the number of pixels in the vertical dimension, $x$ and $y$ are correspondingly the horizontal and vertical pixel coordinate, and $\alpha, \beta$, and $\gamma$ the positive constants. The terms $l_{\mathrm{ri}}, c_{\mathrm{ri}}$, and $f_{\text {ri }}$ stand for the luminance, contrast, and image structure similarity between two images, respectively:

$l_{\mathrm{ri}}=\left(2 \mu_{\mathrm{r}} \mu_{\mathrm{i}}+N 1\right) /\left(\mu_{\mathrm{r}}^{2}+\mu_{\mathrm{i}}^{2}+N 1\right)$

$c_{\mathrm{ri}}=\left(2 \sigma_{\mathrm{r}} \sigma_{\mathrm{i}}+N 2\right) /\left(\sigma_{\mathrm{r}}^{2}+\sigma_{\mathrm{i}}^{2}+N 2\right)$

$f_{\mathrm{ri}}=\left(\operatorname{cov}_{\mathrm{ri}}+N 3\right) /\left(\sigma_{\mathrm{r}} \sigma_{\mathrm{i}}+N 3\right)$,

where $\mu$ is the pixel mean of an image, $\sigma$ the pixel value standard deviation, cov the covariance, and $N 1, N 2$, and $N 3$ are constants (Renieblas et al., 2017). The indices $r$ and i stand for reference and sample image, respectively. SSIM was calculated using the MATLAB ${ }^{\circledR}$ function ssim.

MSE and PSNR are standard tools for the assessment of similarity between a pair of images and were computed using MATLAB ${ }^{\circledR}$ functions immse and psnr, respectively. Mean squared error is defined as (Gonzalez and Woods, 1992)

$\mathrm{MSE}=\frac{1}{X Y} \sum_{x=1}^{X} \sum_{y=1}^{Y}\left[g_{\mathrm{r}}(x, y)-g_{\mathrm{i}}(x, y)\right]$,

where $g_{\mathrm{r}}$ is the reference image and $g_{\mathrm{i}}$ is the sample image.

PSNR is calculated as (Gonzalez and Woods, 1992)

PSNR $=-10 \log _{10} \frac{\mathrm{MSE}}{S^{2}}$,

where $S^{2}$ is the maximum pixel value.

\section{Appendix D: Georeferencing}

An inverse affine transformation was calculated for the reference frame of each flight to transfer it to the UTM35 coordinates, with the RMSE of fits equaling 0.32, 0.11, 0.48, and $0.43 \mathrm{~m}$ for flights $1-4$, respectively:

$$
\begin{aligned}
\mathrm{UTM}_{\text {lat }} & =\frac{-\left(a \cdot f-a \cdot y_{\mathrm{pix}}-c \cdot d+d \cdot x_{\mathrm{pix}}\right)}{(a \cdot e-b \cdot d)} \\
\mathrm{UTM}_{\mathrm{lon}} & =\frac{\left(-e \cdot c+e \cdot x_{\mathrm{pix}}+f \cdot b-b \cdot y_{\mathrm{pix}}\right)}{(a \cdot e-b \cdot d)},
\end{aligned}
$$

where $a, b, c, d, e$, and $f$ are the parameters of affine transform, $x_{\text {pix }}$ and $y_{\text {pix }}$ the (righthanded) coordinates of a pixel in an original image, and $\mathrm{UTM}_{\text {lat }}$ and $\mathrm{UTM}_{\mathrm{lon}}$ the UTM northing and easting of a transformed image. The fit parameters in Eqs. (D1) and (D2) can be found in Table D1. As the resolution of the original images was about $0.6 \mathrm{~m}$, for convenience, a $1 \mathrm{~m}$ resolution was used for the UTM grid onto which the images were transferred. As the images were coregistered in Step 3 (Sect. 2.2.3), the same set of parameters was applied to georeference each subsequent image of a sequence.

Table D1. Affine transform coefficients applied to the registered thermogram sequences of each flight along with the root mean squared values (RMSE).

\begin{tabular}{lrrrr}
\hline & Flight 1 & Flight 2 & Flight 3 & Flight 4 \\
& 6 August & 6 August & 28 August & 28 August \\
\hline$a$ & -0.2536 & -0.2490 & -0.1683 & -0.2573 \\
$b$ & -0.6017 & -0.6057 & -0.6430 & -0.6124 \\
$c$ & 352435.98 & 352431.41 & 352404.79 & 352425.36 \\
$d$ & 0.6009 & 0.6048 & 0.6430 & 0.6124 \\
$e$ & -0.2533 & -0.2492 & -0.1659 & -0.2576 \\
$f$ & 6858603.63 & 6858595.52 & 6858569.46 & 6858599.99 \\
RMSE (m) & 0.32 & 0.11 & 0.48 & 0.43 \\
\hline
\end{tabular}


Code availability. The codes are freely available at https://doi.org/ 10.5281/zenodo.4019155 (Alekseychik, 2020a).

Data availability. The data are freely available at https://doi.org/ 10.5281/zenodo.4019321 (Alekseychik, 2020b).

Video supplement. The visualizations of turbulence for the four flights are available at https://doi.org/10.5281/zenodo.4019175 (Alekseychik, 2020c). Additionally, the videos may be watched on YouTube: Flight 1, 6 August: https://youtu.be/UwN8rFQ3Y0E (last access: 17 May 2021); Flight 2, 6 August: https://youtu. be/UeNU8lq7krY (last access: 17 May 2021); Flight 1, $28 \mathrm{Au}-$ gust: https://youtu.be/K4ahj0EtrWM (last access: 17 May 2021); Flight 2, 28 August: https://youtu.be/jgC2GDptLtU (last access: 17 May 2021).

Author contributions. PA conceived the study, conducted the measurements and their analyses, wrote the text, and produced the figures. GK helped develop the theoretical and analytical framework and contributed to the text. IK helped develop the image processing methods and contributed to the text. SL made major contributions throughout the study including theoretical and analytical methods and writing parts of the text.

Competing interests. The authors declare that they have no conflict of interest.

Acknowledgements. Pavel Alekseychik and Samuli Launiainen acknowledge the support of the projects CLIMOSS (Climate impacts of boreal bryophytes - from functional traits to global models), funded by the Academy of Finland, Decision no. 296116, and SOMPA (Novel soil management practices - key for sustainable bioeconomy and climate change mitigation), funded by the Strategic Research Council at the Academy of Finland, Decision no. 312912. Timo Vesala (INAR, University of Helsinki) is gratefully acknowledged for providing the drone and thermal camera used in this study.

Financial support. This research has been supported by the Academy of Finland (Project CLIMOSS (Climate impacts of boreal bryophytes - from functional traits to global models), Decision no. 296116; and Project SOMPA (Novel soil management practices - key for sustainable bioeconomy and climate change mitigation), Decision no. 312912).

Open-access funding was provided by the Helsinki University Library.

Review statement. This paper was edited by Marcos Portabella and reviewed by two anonymous referees.

\section{References}

Adrian, R. J.: Hairpin vortex organization in wall turbulence, Phys. Fluids, 19, 041301, https://doi.org/10.1063/1.2717527, 2007.

Albertson, J. D., Parlange, M. B., Katul, G. G., Chu, C., Stricker, H., and Tyler, S.: Sensible heat flux from arid regions: A simple flux-variance method, Water Resour. Res., 31, 969-973, 1995.

Alekseychik, P. K.: Codes to analyze atmospheric boundary layer turbulence based on thermal videos (Version 1.0), Zenodo [code], https://doi.org/10.5281/zenodo.4019155, 2020a.

Alekseychik, P. K.: Georeferenced drone thermal video and related data, recorded on 6 and 28 August 2019 in Siikaneva peatland (Version 1.0), Zenodo [data set], https://doi.org/10.5281/zenodo.4019321, 2020b.

Alekseychik, P. K.: Turbulence on natural peatland surface visualized using thermal videos (Version 1.0), Zenodo, https://doi.org/10.5281/zenodo.4019175, 2020c.

Alekseychik, P. K., Korrensalo, A., Mammarella, I., Vesala, T., and Tuittila, E. S.: Relationship between aerodynamic roughness length and bulk sedge leaf area index in a mixed-species boreal mire complex, Geophys. Res. Lett., 44, 5836-5843, https://doi.org/10.1002/2017GL073884, 2017a.

Alekseychik, P., Mammarella, I., Karpov, D., Dengel, S., Terentieva, I., Sabrekov, A., Glagolev, M., and Lapshina, E.: Net ecosystem exchange and energy fluxes measured with the eddy covariance technique in a western Siberian bog, Atmos. Chem. Phys., 17, 9333-9345, https://doi.org/10.5194/acp-179333-2017, 2017b.

Antonia, R. A., Chambers, A. J., Friehe, C. A., and Van Atta, C. W. Temperature Ramps in the Atmospheric Surface Layer, J. Atmos. Sci. 36, 99-108, 1979.

Christen, A. and Voogt, J.: Linking atmospheric turbulence and surfate temperature fluctuations in a street canyon, in: Proceedings of the Seventh International Conference on Urban Climate, 29 June-3 July 2009, Yokohama, Japan, 2009.

Christen, A., Meier, F., and Scherer, D.: High-frequency fluctuations of surface temperatures in an urban environment, Theor. Appl. Climatol., 108, 301-324, 2012.

Chudnovsky, A., Ben-Dor, E., and Saaroni, H.: Diurnal thermal behavior of selected urban objects using remote sensing measurements, Energ. Buildings, 36, 1063-1074, https://doi.org/10.1016/j.enbuild.2004.01.052, 2004.

Davison, D. S.: The horizontal cross-sectional shape of convective plumes, Q. J. Roy. Meteor. Soc., 101, 463-473, 1975.

Drobinski, P., Carlotti, P., Newsom, R. K., Banta, R. M., Foster, R. C., and Redelsperger, J.: The structure of the near-neutral atmospheric surface layer, J. Atmos. Sci., 61, 699-714, https://doi.org/10.1175/15200469(2004)061<0699:TSOTNA>2.0.CO;2, 2004.

Dugdale, S. J., Kelleher, C., Malcolm, I. A., Caldwell, S., and Hannah, D. M.: Assessing the potential of dronebased thermal infrared imagery for quantifying river temperature heterogeneity, Hydrol. Process. 33, 1152-1163, https://doi.org/10.1002/hyp.13395, 2019.

Fang, J. and Porté-Agel, F.: Large-eddy simulation of very-largescale motions in the neutrally stratified atmospheric boundary layer, Bound.-Lay. Meteorol., 155, 397-416, 2015.

Frisch, A. S. and Businger, J. A.: A Study of Convective Elements in the Atmospheric Boundary Layer, Bound.-Lay. Meteorol., 3, 301-328, 1973. 
Ganapathisubramani, B., Longmire, E. K., and Marusic, I.: Characteristics of vortex packets in turbulent boundary layers, J. Fluid Mech. 478, 35-46, 2003.

Garai, A. and Kleissl, J.: Air and surface temperature coupling in the convective atmospheric boundary layer, J. Atmos. Sci., 68, 2945-2954, 2011.

Garai, A. and Kleissl, J.: Interaction between coherent structures and surface temperature and its effect on ground heat flux in an unstably stratified boundary layer, J. Turbul., 14, 1-23, https://doi.org/10.1080/14685248.2013.806812, 2013.

Gonzalez, R. C. and Woods, R. E.: Digital Image Processing, Addison-Wesley, New York, USA, 1992.

Guala, M., Hommema, S. E., and Adrian, R. J.: Large-scale and very-largescale motions in turbulent pipe flow, J. Fluid Mech., $554,521-542,2006$

Hoyano, A., Asano, K., and Kanamaru, T.: Analysis of the sensible heat flux from the exterior surface of buildings using time sequential thermography, Atmos. Environ., 33, 3941-3951, 1999.

Inagaki, A., Kanda, M., Onomura, S., and Kumemura, H.: Thermal Image Velocimetry, Bound.-Lay. Meteorol., 149, 1-18, https://doi.org/10.1007/s10546-013-9832-z, 2013.

Jeong, J., Hussain, F., Schoppa, W., and Kim, J.: Coherent structure near the wall in a turbulent channel flow, J. Fluid Mech., 332, 185-214, 1997.

Kaimal, J. C.: Translation Speed of Convective Plumes in the ASL, Q. J. Roy. Meteor. Soc., 100, 46-52 1974.

Kaimal, J. C. and Businger, J. A.: Case Studies of a Convective Plume and a DustDevil, J. Appl. Meteorol., 9, 612-620, 1970.

Kaimal, J. C., Wyngaard, J. C., HAugusten, D. A., Cotè, O. R., Izumi, Y., CAugusthy, S. J., and Readings, C. J.: Turbulence structure in theconvective boundary layer, J. Atmos. Sci., 33, 2152-2169, 1976.

Katul, G. G., Schieldge, J., Hsieh, C. I., and Vidakovic, B.: Skin temperature perturbations induced by surface layer turbulence above a grass surface, Water Resour. Res., 34, 1265-1274, 1998.

Khalsa, J. S.: Surface-layer intermittency investigated with conditional sampling, Bound.-Lay. Meteorol., 19, 135-153, 1980.

Kim, K. C. and Adrian, R. J.: Very large-scale motion in the outer layer, Phys. Fluids, 11, 417, https://doi.org/10.1063/1.869889, 1999.

Kline, S. J., Reynolds, W. C., Schraub, R. A., and Runstadler, P. W.: The structure of turbulent boundary layers, J. Fluid Mech., 30, 741-773, 1967.

Kormann, R. and Meixner, F. X.: An Analytical Footprint ModelFor Non-Neutral Stratification, Bound.-Lay. Meteorol., 99, 207-224, https://doi.org/10.1023/a:1018991015119, 2001.

Laima, S., Ren, H., Li, H., and Ou, J.: Numerical Simulation of Coherent Structures in the Turbulent Boundary Layer under Diferent Stability Conditions, Energies, 13, 1068, https://doi.org/10.3390/en13051068, 2020.

Margairaz, F., Pardyjak, E. R., and Calaf, M.: Surface thermal heterogeneities and the atmospheric boundary layer: the relevance of dispersive fluxes, Bound.-Lay. Meteorol, 175, 369-395, 2020.

Mattes, D., Haynor, D. R., Vesselle, H., Lewellen, T., and Eubank. W. Non-rigid multimodality image registration, in: Proceedings of SPIE 4322, Medical Imaging 2001: Image Processing, 3 July 2001, San Diego, CA, USA, 1609-1620, https://doi.org/10.1117/12.431046, 2001.
Meier, F., Scherer, D., Richters, J., and Christen, A.: Atmospheric correction of thermal-infrared imagery of the 3-D urban environment acquired in oblique viewing geometry, Atmos. Meas. Tech., 4, 909-922, https://doi.org/10.5194/amt-4-909-2011, 2011.

Morrison, T. J., Calaf, M., Fernando, H. J. S., Price, T. A., and Pardyjak, E. R.: A methodology for computing spatiallyand temporally varying surface sensible heat flux from thermal imagery, Q. J. Roy. Meteor. Soc., 143, 2616-2624, https://doi.org/10.1002/qj.3112, 2017.

Newsom, R., Calhoun, R., Ligon, D., and Allwine, J.: Linearly Organized Turbulence Structures Observed Over a Suburban Area by Dual-Doppler Lidar, Bound.-Lay. Meteorol., 127, 111-130, 2008.

Owen, P. R. and Thompson, W. R.: Heat Transfer Across Rough Surfaces, J. Fluid Mech., 15, 321-334, 1963.

Paw U, K. T., Qiu, J., Su, H. B., Watanabe, T., and Brunet, Y.: Surface renewal analysis: a new method to obtain scalar ?uxes without velocity data, Agr. Forest Meteorol., 74, 119-137, 1995.

Priestley, C. H. B.: Turbulent Transfer in the Lower Atmosphere, University of Chicago Press, Chicago, USA, 130 pp., 1959.

Renieblas, G. P., Nogués, A. T., González, A. M., Gómez-Leon, N., and del Castillo, E. G.: Structural similarity index family for image quality assessment in radiological images, J. Med. Imag., 4, 035501, https://doi.org/10.1117/1.JMI.4.3.035501, 2017.

Salesky, S. T., Katul, G. G., and Chamecki, M.: Buoyancy effects on the integral lengthscales and mean velocity profile in atmospheric surface layer flows, Phys. Fluids, 25, 105101, https://doi.org/10.1063/1.4823747, 2013.

Stull, R. B.: An Introduction to Boundary Layer Meteorology, in: Atmospheric Sciences Library, Springer, the Netherlands, https://doi.org/10.1007/978-94-009-3027-8, 2011.

Styner, M., C. Brechbuehler, G. Székely, and G. Gerig: Parametric estimate of intensity inhomogeneities applied to MRI, IEEE T. Med. Imaging, 19, 153-165, 2000.

Sugawara, H., Narita, K., and Mikami, T.: Estimation of effective thermal property parameter on a heterogeneous urban surface, J. Meteorol. Soc. Jpn., 79, 1169-1181, 2001.

Taylor, R. J.: Thermal Structures in the Lowest Layers of the Atmosphere, Aust. J. Phys. 11, 168-176, 1958.

Thielicke, W. and Stamhuis, E. J.: PIVlab - Towards UserFriendly, Affordable and Accurate Digital Particle Image Velocimetry in MATLAB, J. Open Res. Software, 2, e30, https://doi.org/10.5334/jors.bl, 2014.

Tomkins, C. D. and Adrian, R. J.: Spanwise structure and scale growth in turbulent boundary layers, J. Fluid Mech., 490, 37-74, 2003.

Vogt, R.: Visualisation of turbulent exchange using a thermal camera, 18th symposium on boundary layer and turbulence, 913 June 2008, Stockholm, Sweden, Paper no. 8B.1, 2008.

Webb, E. K.: Convection mechanisms of atmospheric heat transfer from surface to global scales, in: Proc. Second Australasian Conf. on Heat and Mass Transfer, February 1977, Sydney, Australia, NTIS 77N30685/2, 523-539, 1977.

Wilczak, J. M. and Tillman, J. E.: The three-dimensional structure of convection in the atmospheric surface layer, J. Atmos. Sci., 37, 2424-2443, 1980. 